\title{
An Efficient Radio Access Resource Management Scheme Based on Priority Strategy in Dense mmWave Cellular Networks
}

\author{
Jinsong Gui $\mathbb{D}$ and Jianglin Liu \\ School of Computer Science and Engineering, Central South University, Changsha 410083, China \\ Correspondence should be addressed to Jinsong Gui; jsgui06@163.com
}

Received 20 June 2020; Revised 15 August 2020; Accepted 5 September 2020; Published 15 September 2020

Academic Editor: Stefano Savazzi

Copyright ( 2020 Jinsong Gui and Jianglin Liu. This is an open access article distributed under the Creative Commons Attribution License, which permits unrestricted use, distribution, and reproduction in any medium, provided the original work is properly cited.

\begin{abstract}
In millimeter wave (mmWave) communication systems, beamforming-enabled directional transmission and network densification are usually used to overcome severe signal path loss problem and improve signal coverage quality. The combination of directional transmission and network densification poses a challenge to radio access resource management. The existing work presented an effective solution for dense mmWave wireless local area networks (WLANs). However, this scheme cannot adapt to network expansion when it is applied directly to dense mmWave cellular networks. In addition, there is still room for improvement in terms of energy efficiency and throughput. Therefore, we firstly propose an efficient hierarchical beamforming training (BFT) mechanism to establish directional links, which allows all the small cell base stations (SBSs) to participate in the merging of training frames to adapt to network expansion. Then, we design a BFT information-aided radio access resource allocation algorithm to improve the downlink energy efficiency of the entire mmWave cellular network by reasonably selecting beam directions and optimizing transmission powers and beam widths. Simulation results show that the proposed hierarchical BFT mechanism has the smaller overhead of BFT than the existing BFT mechanism, and the proposed BFT information-aided radio access resource allocation algorithm outperforms the existing corresponding algorithm in terms of average energy efficiency and throughput per link.
\end{abstract}

\section{Introduction}

The growing popularity of the mobile Internet and the Internet of things [1-3] as well as the increasing perceptual services [4-6] has led to a continued high demand for network capacity, in which more spectrum is needed to support [7]. In order to relieve the pressure of spectrum resource supply, various schemes for efficiently exploiting spectrum have been proposed [8-11], but they still cannot satisfy people's thirst for high capacity and massive connectivity $[12,13]$. Since spectrum resources in the traditional cellular frequency bands have dried up, millimeter wave (mmWave) band has become a new source of spectrum supply [14], which has rich spectrum resources and thus can support the wireless transmissions with high data rate. Now, mmWave communication technology has been applied to both the fifth-generation (5G) cellular systems and wireless local area networks (WLANs) (e.g., IEEE 802.11ad and IEEE 802.11ay)
[15]. However, before making full use of its abundant spectral resources, it is necessary to overcome its inherent defects (e.g., severe path loss, high oxygen absorption, and blockages). Fortunately, the short mmWave wavelength facilitates the deployment of a large number of antennas on a smallsized terminal, and thus, it is also helpful to improve communication quality or extend communication distance by using antenna array technology and directional transmission.

In addition, by densely deploying base stations, the average communication distance can be reduced, and the signal coverage quality can also be improved because the probability of blockages goes down [16]. However, the combination of directional transmission and network densification will create new difficulties for radio access resource management, where improper mmWave beam allocation will lead to nonnegligible interferences between communication nodes. The authors of [17] believe that the beam management and interference coordination (BM-IC) in dense mmWave network is 
very challenging, while some researchers focus on the evolution of WLANs and the corresponding resource management, beam management, interference management, and mobility management [18-20].

Unlike the above studies that focus on the sum data rate improvement of the entire mmWave WLAN by proper mmWave beam allocation, we focus on the energy efficiency improvement of the entire mmWave cellular network, since the energy consumption problem becomes more prominent in high-capacity network applications. The main contributions of this paper mainly include two aspects. Firstly, in order to reduce the overhead of beamforming training (BFT) in a dense mmWave cellular network, inspired by the BFT mechanism in [19], we propose an efficient hierarchical BFT mechanism to establish directional links. Secondly, we model the radio access resource management for downlink communication in the dense mmWave cellular network as an optimization problem and design a hierarchical BFT informationaided radio access resource allocation algorithm to improve the downlink energy efficiency of the entire mmWave cellular network by reasonably selecting beam directions and optimizing transmission powers and beam widths.

The remainders of this paper are organized as follows. In Section 2, we review related work with respect to radio resource management (RRM). The network model and beamforming training are described in Section 3, while the problem formulation is given in Section 4. The design details regarding the proposed scheme are proposed in Section 5. The simulation results are evaluated in Section 6. Finally, the conclusion of this paper is summarized in Section 7.

\section{Related Work}

In this section, we mainly introduce the existing studies regarding RRM. For WLANs, some researchers think that distributed network architecture makes RRM very inefficient while centralized network architecture is helpful to achieve more efficient RRM [21-23]. Unlike these schemes that all work at low-frequency band and adopt the wired links between controller and access points (APs), in dense mmWave WLANs, a controller is used for managing and controlling multiple APs by directional mmWave links [24, 25]. The authors of [18-20] believed that cloud radio access network- (C-RAN-) based centralized control mode should be applied to WLANs. Therefore, efficient and smart algorithms are needed for mmWave beam management and interference management in WLANs. For example, a deep learning-based BM-IC method in dense mmWave WLANs was proposed in [19]. Based on the centralized architecture, the authors first proposed that an efficient BFT mechanism is to reduce the BFT overhead. Then, they formulated the BM-IC as an optimization problem and designed a BFT information-aided BM-IC algorithm to solve it. Finally, they trained a deep neural network (DNN) model to approximate the proposed algorithm by using the training data obtained from the proposed algorithm.

Since cellular networks also exploit mmWave frequencies to increase network capacity, some literatures reviewed the RRM studies on dense mmWave cellular networks. The authors of [26] reviewed the mmWave beam management procedures included in the 3GPP New Radio (NR) specifications based on both standalone and nonstandalone architectures and provided the performance comparison among different schemes and the design insights into the most important parameters associated with the beam management frameworks. The authors of [27] reviewed the existing measurement techniques for beam and mobility management in mmWave cellular networks and proposed the insights into the design of accurate, reactive, and robust control layer procedures (e.g., initial access, handover, and beam tracking). The authors of [28] reviewed the existing economic and pricing approaches for addressing resource management issues in the $5 \mathrm{G}$ wireless networks including user association, spectrum allocation, and interference and power management.

Some studies focused on BM-IC problem in dense mmWave cellular networks. The authors of [16] explored the power control and transmission duration allocation problem in self-backhauling dense mmWave cellular networks. The authors of [17] explored the potential gain of ultradensification in mmWave cellular networks and proposed a large-scale channel state information- (CSI-) based interference coordination approach to solve the cochannel interference. The authors of [29] explored the beam pair selection problem in heterogeneous multibeam cloud radio access network architecture, which is aimed at improving the mmWave cellular network performance. The authors of [30] studied the joint power control and user association problem in mmWave cellular communications with directional transmission and dense deployment and proposed a reinforcement learning framework to address this problem. The authors of [31] explored resource allocation problem in $5 \mathrm{G}$ mmWave small cell systems, presented a hybrid beamforming structure for small cell base station (SBS), and formulated the joint allocation of the beam and transmission power on the subcarriers into a mixed integer nonlinear programming problem and decomposed it into the beam selection and power allocation subproblems.

Exploiting the benefits from dense mmWave networks with multiple SBSs and many user equipment (UE), the authors of [32] discussed an approach for jointly optimizing SBS-UE association and power allocation, which can maximize the system energy efficiency (EE) while guaranteeing the quality of service (QoS) constraints for each UE. However, the mechanisms mentioned above do not consider the BFT problem in dense mmWave cellular networks. Since users and base stations need to align their beams during both initial access and data transmissions to ensure their end-toend performance and quality of service, an effective BFT mechanism is needed to ensure the accuracy of beam selection and reduce the delay in performing initial access or updating beam pair in dense mmWave cellular networks.

\section{Network Model and Beamforming Training}

3.1. Network Model. As shown in Figure 1, there are I SBSs overlapped in a micro cell and $J$ UEs randomly distributed in the same macro cell, where a micro base station (MBS) is located at the center of this micro cell. We denote the set of 


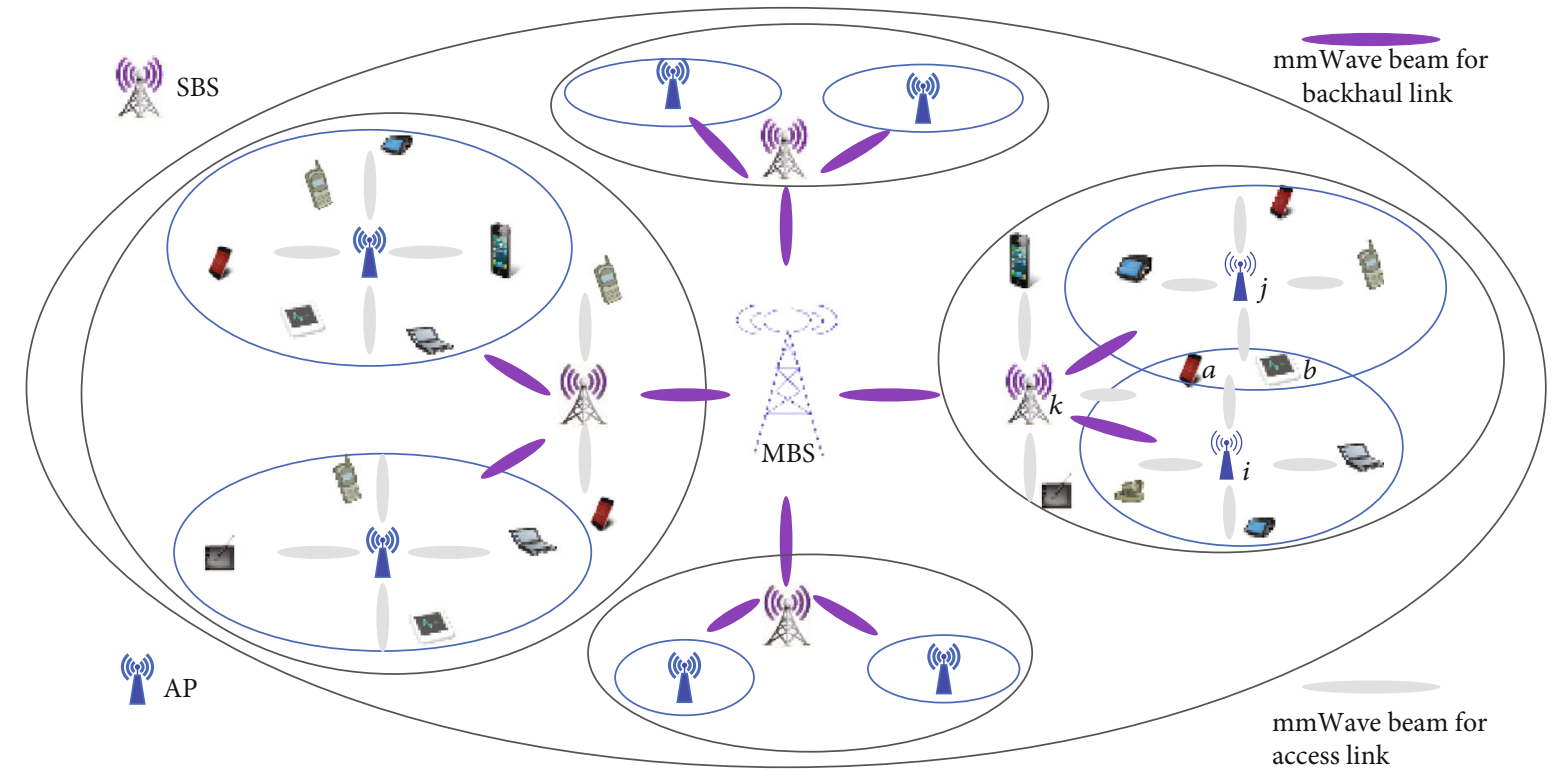

Figure 1: A dense mmWave cellular network.

$I$ SBSs as $\mathbb{Q}=\{\operatorname{SBS} 1, \cdots, \operatorname{SBS} i, \cdots, \operatorname{SBS} I\}$ and the set of $J$ UEs as $\mathbb{N}=\{\mathrm{UE} 1, \cdots, \mathrm{UE} j, \cdots, \mathrm{UE} J\}$, respectively. Also, there are $K$ APs overlapped in the $I$ small cells. We mark the set of $K$ APs as $\mathbb{C}=\{\operatorname{AP} 1, \cdots, \operatorname{AP} k, \cdots, \operatorname{AP} K\}$.

In this paper, a SBS is indirectly connected to the core network via the MBS in a wireless mode (e.g., mmWave communication) or directly connected to the core network in a wired mode (e.g., optical fiber), while an AP is only indirectly connected to the core network via a SBS in a mmWave communication mode.

Each AP can schedule a set of mmWave frequency resources and allocate them to the UEs associated by it in an orthogonal mode. The same mmWave frequency resources can be reused by the other APs. Also, these resources are shared among the MBS and SBSs in an underlay mode for the purpose of providing wireless access service.

Each UE selects one of the APs (or SBSs) around itself to perform channel access and data transmission through directional link (i.e., the gray beams in Figure 1), where the MBS is not selected for direct access by any UE, so it can focus on providing backhaul connection for SBSs and control plane information transmission. When the MBS and SBSs provide wireless backhaul service, they will use dedicated mmWave frequency bands to avoid interfering with each other. Based on the assumption of unrestricted backhaul capacity, we only focus on radio access resource management problem for downlink unicast communications in a dense mmWave cellular network.

To facilitate the evaluation of energy efficiency for dense mmWave cellular networks, we adopt the commonly used switch-based analog beam pattern [33]. The normalized beamforming gain $G$ is as follows.

$$
G(\theta, \phi)=\left\{\begin{array}{l}
\frac{2 \pi-(2 \pi-\theta) \partial}{\theta}, \text { if }|\phi| \leq \frac{\theta}{2} \\
\partial, \text { otherwise, }
\end{array}\right.
$$

where $\theta$ is the beam width of the main lobe in radian, $\phi$ is the beam offset angle to the main lobe in radian, and $\partial$ is the gain of the side lobe and $0<\partial \ll 1$.

For analytical tractability, we assume that the number of beams of each of MBS, SBS, AP, and UE is limited, and each beam covers a unique direction in a nonoverlapping way. Therefore, the number of beams of each of MBS, SBS, AP, and UE is denoted as $n_{\text {beam }}$, which is estimated as follows.

$$
n_{\text {beam }}=\left\lfloor\frac{2 \pi}{\theta_{\max }}\right\rfloor,
$$

where $\theta_{\max }$ is the maximum beam width of MBS, SBS, AP, and UE.

\subsection{Efficient Beamforming Training Mechanism for Dense} mmWave Cellular Network. As shown in Figure 1, when a $\mathrm{UE}$ is covered by multiple APs (e.g., UE $a$ is covered by AP $i$ and AP j), in order to allow the UE to select a suitable AP (e.g., AP i) among all APs for access in terms of smaller interference, the UE should perform beamforming training (BFT) with its surrounding APs in advance.

Since the BFT mechanism in IEEE 802.11ad/ay only considers the BFT between one AP and one wireless terminal or between one AP and multiple wireless terminals, it will generate serious collision problem if multiple APs are allowed to perform BFT simultaneously [18]. When this BFT mechanism is directly applied to dense mmWave networks, all APs have to perform BFT one by one to avoid collision, which makes BFT be inefficient. Therefore, the authors in [19] proposed an efficient BFT mechanism to coordinate the BFT processes of every AP and wireless terminals so that many duplicated BFT processes can be merged. However, the merge decision is made by a single node (e.g., a MBS), which is difficult to adapt to an increasing number of wireless terminals. It may be an effective solution to assign merge decision 


\begin{tabular}{|c|c|c|c|}
\hline $\begin{array}{c}\text { Transmitting training } \\
\text { request }\end{array}$ & $\begin{array}{c}\text { Waiting for training } \\
\text { response }\end{array}$ & $\begin{array}{c}\text { Handling response } \\
\text { conflicts }\end{array}$ & $\begin{array}{c}\text { Announcing training } \\
\text { results }\end{array}$ \\
\hline
\end{tabular}

FIgURE 2: The time slot structure of the training initiator.

\begin{tabular}{|c|c|c|c|c|}
\hline INI_ID & REQ_NUM & REQ_BEAM_ID & NARS & REQ_TX_POW \\
\hline
\end{tabular}

(a) The training request beacon frame

\begin{tabular}{|l|l|l|l|l|}
\hline REP_ID & ID_NUM & REP_BEAM_ID & PLV & REP_TX_POW \\
\hline
\end{tabular}

(b) The training response beacon frame

\begin{tabular}{|l|l|l|l|l|}
\hline INI_ID & REP_ID & REQ_BEAM_ID & REP_BEAM_ID & SNFB \\
\hline
\end{tabular}

(c) The beacon frame of announcing training results

Figure 3: The structure of beacon frames.

tasks to multiple nodes (e.g., SBSs). Therefore, the basic idea of our proposed efficient hierarchical BFT mechanism is shown as below.

Step 1. (BFT between the MBS and its surrounding SBSs). This step is initiated by the MBS, and the time slot structure of its training process is shown in Figure 2.

In the transmitting training request phase, the MBS simultaneously transmits the training request beacon frames in all its beams to start the training process between the MBS and SBSs.

In the waiting for training response phase, each SBS that receives request beacon frame randomly selects a response slot to simultaneously transmit its training response beacon frames in all its beams.

If the response beacon frame conflict (that refers to the occurrence of more than one response beacon frame on the same beam at the MBS side) does not occur, the handling response conflicts phase can be ignored. Otherwise, the MBS simultaneously retransmits the training request beacon frames in the beams where there are conflicting events. In these retransmitted frames, the number of response slots is doubled to reduce the probability of conflict.

In the announcing training results phase, the MBS transmits the beacon frames for announcing training results to the SBSs that have successfully sent the training response beacon frames through the best beams estimated by it according to the information of the training response beacon frames.

The structure of beacon frames used in the above training process is shown in Figure 3. In the training request beacon frame, INI_ID represents the initiator's identifier (e.g., the MBS); REQ_NUM denotes the serial number of this frame; REQ_BEAM_ID is the serial number of the beam in which this frame is transmitted; NARS records the number of allowed response slots; REQ_TX_POW is the power used to transmit this frame.

In the training response beacon frame, REP_ID represents the responder's identifier (e.g., a SBS); INI_NUM denotes a pair of values, which comes from the INI_ID and REQ_NUM of the training request beacon frame received by this responder; REP_BEAM_ID is the serial number of the beam in which this frame is transmitted; PLV denotes the path loss value, which is estimated according to the REQ_TX_POW in the training request beacon frame received by this responder and the receiving signal strength perceived by this responder; REP_TX_POW is the power used to transmit this frame, which facilitates the initiator to estimate the path loss value.

In the beacon frame of announcing training results, SNFB is the serial number of frequency band, that is to say, it denotes the channel between the initiator (i.e., INI_ID) and the responder (i.e., REP_ID), where the initiator's beam (i.e., REQ_BEAM_ID) can cover the responder and the responder's beam (i.e., REP_BEAM_ID) can cover the initiator. This pair of beams should minimize the weighted sum of the two-way path loss values between the initiator and the responder. Otherwise, this pair of beams will not be placed in this frame. If different frequency bands are used on the same pair of beams, concurrent transmission can be achieved without conflict.

Step 2. (BFT between every SBS and its surrounding APs). This step is initiated by every SBS, and the time slot structure of its training process is similar to that of Figure 2. Since all SBSs must perform BFT one by one to avoid collision, there may be the case where an AP is associated with more than one SBS. Therefore, each SBS firstly transmits its built beacon frame of announcing training results to the MBS via mmWave link or optical fiber. Based on the results reported by all SBSs, the MBS performs comparative analysis and produces results and then feedbacks the APs with which each SBS should associate. After that, each SBS sends each expected associated AP the corresponding beacon frame.

Step 3. (BFT between every AP and its surrounding UEs). This step is initiated by every AP, and the time slot structure 
of its training process is also similar to that of Figure 2. Similar to Step 2, since all APs must perform BFT one by one to avoid collision, there may be the case where an UE is associated with more than one AP. Therefore, each AP firstly transmits its built beacon frame of announcing training results to its associated SBS (that is determined in Step 2). Based on the results reported by all the APs associated by a SBS, this SBS performs comparative analysis and produces results and then feedbacks the UEs with which each AP should associate. After that, each AP sends each expected associated UE the corresponding beacon frame.

By Steps 1-3, we only finish the transmitting BFT of SBSs, APs, and UEs instead of their receiving BFT. Due to the reciprocity of directional beams in mmWave band, both transmitting BFT and receiving BFT can be done in a similar way [15]. In order to finish the receiving BFT, the beam refinement protocol phase in IEEE 802.11ad/ay can be performed by applying the similar modifications of Steps 1-3. For more details (e.g., training processes and specific frame formats), please refer to [18].

3.3. Performance Analysis of Beamforming Training. Without loss of generality, we assume that each of SBSs, APs, and UEs can generate $n_{\text {beam }}$ beams. Therefore, the total number of training frames required for our proposed efficient hierarchical BFT mechanism should be at least as follows.

$$
\begin{aligned}
n_{\text {frame }}= & \left(n_{\text {beam }}+I_{m} \bullet n_{\text {beam }}+I_{m}\right)+I \bullet n_{\text {beam }} \\
& +\sum_{k=1}^{K}\left(n_{\text {beam }}+2 I_{k}+1\right)+K \bullet n_{\text {beam }}+\sum_{j=1}^{J}\left(n_{\text {beam }}+2 K_{j}+1\right) .
\end{aligned}
$$

If the BFT mechanism in [19] is used in this paper, the total number of training frames should be at least as follows.

$$
\begin{aligned}
n_{\text {frame }}^{6}= & n_{\text {beam }}+(K+I) \bullet n_{\text {beam }}+(K+I)+(K+I+1) \bullet n_{\text {beam }} \\
& +J \bullet\left(n_{\text {beam }}+2(K+I)+1\right) .
\end{aligned}
$$

Therefore, the total number of training frames saved by our proposed efficient hierarchical BFT mechanism is estimated by the following formula.

$n_{\text {save }}=n_{\text {frame }}^{6}-n_{\text {frame }} \geq\left(I-I_{m}\right) \cdot\left(n_{\text {beam }}+1\right)+n_{\text {beam }}+2 I \bullet(J-K)$.

Compared to the BFT mechanism in [19], our proposed efficient hierarchical BFT mechanism can reduce BFT overhead. More importantly, the proposed BFT mechanism will be more efficient when the number of UEs (i.e., $J$ ) is much larger than that of APs (i.e., $K$ ).

The proof of equation (3) is given as follows. When the MBS performs the BFT with $I_{m}$ SBSs, where we assume that $I_{m}$ SBSs can be directly connected with the MBS via mmWave links and $I_{m} \leq I$, it should transmit $n_{\text {beam }}$ training frames in its $n_{\text {beam }}$ beams to complete the transmission of training request phase in Step 1. In the waiting for training response phase, each of the $I_{m}$ SBSs should transmit $n_{\text {beam }}$ training frames in a randomly selected response slot. If there is no conflict of response beacon frames, the MBS should transmit $I_{m}$ beacon frames for announcing training results to $I_{m}$ SBSs. Therefore, there are at least $\left(n_{\text {beam }}+I_{m} \bullet n_{\text {beam }}+I_{m}\right)$ training frames required for completing Step 1 in Section 3.2.

For Step 2 in Section 3.2, I SBSs should transmit $n_{\text {beam }}$ training frames one by one to complete the transmission of training request phase. In the waiting for training response phase, each of the $K$ APs (e.g., AP $k, k \in\{1,2, \cdots, K\}$ ) should transmit $n_{\text {beam }}$ training frames in a randomly selected response slot. After the AP $k$ transmits $n_{\text {beam }}$ training frames, $I_{k}\left(I_{k} \leq I\right)$ SBSs that receive the training frames from the AP $k$ should transmit $I_{k}$ beacon frames for announcing training results to the MBS. Soon afterwards, the MBS transmits $I_{k}$ beacon frames for announcing training results to $I_{k}$ SBSs. Finally, the selected SBS (based on the signal-to-noise ratio (SNR) maximization) will transmit the beacon frames for announcing training results to APs. Therefore, $K$ APs require $\sum_{k=1}^{K}\left(n_{\text {beam }}+2 I_{k}+1\right)$ training frames to complete the transmission of response beacon frames in total. Therefore, there are at least $I \bullet n_{\text {beam }}+\sum_{k=1}^{K}\left(n_{\text {beam }}+2 I_{k}+1\right)$ training frames required for completing Step 2 in Section 3.2.

For Step 3 in Section 3.2, $K$ APs should transmit $n_{\text {beam }}$ training frames one by one to complete the transmission of the training request phase. In the waiting for training response phase, each of the $J$ UEs (e.g., UE $j, j \in\{1,2, \cdots, J\}$ ) should transmit $n_{\text {beam }}$ training frames in a randomly selected response slot. After UE $j$ transmits $n_{\text {beam }}$ training frames, $K_{j}$ $\left(K_{j} \leq K\right)$ APs that receive the training frames from UE $j$ should transmit $K_{j}$ beacon frames for announcing training results to their associated SBSs, where they are likely to be associated with several adjacent SBSs, even the same SBS. Soon afterwards, these SBSs transmit $K_{j}$ beacon frames for announcing training results to $K_{j}$ APs. Finally, the selected AP (based on the SNR maximization) will transmit the beacon frames for announcing training results to UEs. Therefore, $J$ UEs require $\sum_{j=1}^{J}\left(n_{\text {beam }}+2 K_{j}+1\right)$ training frames to complete the transmission of response beacon frames in total. Therefore, there are at least $K \bullet n_{\text {beam }}+\sum_{j=1}^{J}\left(n_{\text {beam }}+2 K_{j}+1\right)$ training frames required for completing Step 3 in Section 3.2. According to the results of Steps 1-3, equation (3) is true.

The proof of equation (4) is given as follows. In order to perform the BFT mechanism in [19], we assume that the MBS, I SBSs, and $K$ APs can connect to each other via mmWave links, where the whole training process consists of only two steps. In the first step, the MBS performs the BFT with $I$ SBSs and $K$ APs. Thus, it should transmit $n_{\text {beam }}$ training frames in its $n_{\text {beam }}$ beams to complete the transmission of the training request phase. In the waiting for training response phase, each of the $I$ SBSs and $K$ APs should transmit $n_{\text {beam }}$ training frames in a randomly selected response slot. If there is no conflict of response beacon frames, the MBS should transmit $(K+I)$ beacon frames for announcing training results. Therefore, there are at least $n_{\text {beam }}+(K+I) \bullet$ $n_{\text {beam }}+(K+I)$ training frames required for the first step. 
In the second step, $I$ SBSs and $K$ APs should transmit $n_{\text {beam }}$ training frames one by one to complete the transmission of the training request phase. In the waiting for training response phase, each of the $J$ UEs should transmit $n_{\text {beam }}$ training frames in a randomly selected response slot. Then, $I$ SBSs and $K$ APs should transmit $(K+I)$ beacon frames for announcing training results to the MBS. Soon afterwards, the MBS sends back $(K+I)$ beacon frames for announcing training results. Finally, the selected AP or SBS (based on the SNR maximization) will transmit the beacon frames for announcing training results to UEs. So $J \cdot\left(n_{\text {beam }}+2(K+I)\right.$ $+1)$ training frames are required in the waiting for the training response phase. Therefore, there are at least $(K+I+1) \bullet$ $n_{\text {beam }}+J \cdot\left(n_{\text {beam }}+2(K+I)+1\right)$ training frames required in the second step. According to the results of the first step and the second step, equation (4) is true.

Based on the above analysis, we know that the original two-stage BFT mechanism is based on the assumption that all the nodes can be directly connected to each other. Obviously, this assumption does not always hold in the dense mmWave cellular network scenario, where some nodes may be indirectly connected to each other. If the original twostage BFT mechanism is applied to the dense mmWave cellular network scenario, the number of training frames would be also counted between the nodes that are not directly connected to each other. Therefore, there are more training frames from the perspective of theoretical statistics, which gives the illusion of more BFT overhead since the unnecessary training frames are counted.

Moreover, there are not only APs but also SBSs in the dense mmWave cellular network scenario, and also, there are the more UEs than the dense mmWave WLAN. Therefore, from formula (5), we know that the original two-stage BFT mechanism is really unsuitable for the dense mmWave cellular network scenario. As the foregoing, the original two-stage BFT mechanism relies on a single MBS to merge the duplicated BFT processes to reduce the number of BFT frames. If it is applied to the dense mmWave cellular network scenario, it would make the MBS become a performance bottleneck. By having all the SBS and the MBS work together to merge the duplicated BFT processes, our three-stage BFT mechanism can avoid this performance bottleneck.

\section{Problem Formulation}

In a dense mmWave cellular network, if the beams between UE $j$ and AP $k$ are aligned, according to equation (1), we can obtain the directional transmitting gain $G_{j, k}^{t}$ and directional receiving gain $G_{j, k}^{r}$ as

$$
\left\{\begin{aligned}
G_{j, k}^{t}= & \frac{2 \pi-\left(2 \pi-\theta_{j, k}^{t}\right) \partial}{\theta_{j, k}^{t}} \\
G_{j, k}^{r}= & \frac{2 \pi-\left(2 \pi-\theta_{j, k}^{r}\right) \partial}{\theta_{j, k}^{r}}
\end{aligned}\right.
$$

where $\theta_{j, k}^{t}$ is the beam width of the transmitter and $\theta_{j, k}^{r}$ is the beam width of the receiver. According to $[19,34,35]$, the channel between UE $j$ and AP $k$ is given by

$$
h_{j, k}(\tau)=\sum_{\mathrm{ph}=0}^{\mathrm{PH}} G_{j, k}^{t,(\mathrm{ph})} G_{j, k}^{r,(\mathrm{ph})} \chi_{j, k}^{c,(\mathrm{ph})} \delta\left(\tau-\tau_{j, k}^{(\mathrm{ph})}\right),
$$

where $\mathrm{PH}$ is the number of paths and the superscript "(ph)" represents the ph-th path. $G_{j, k}^{t,(\mathrm{ph})}$ and $G_{j, k}^{r,(\mathrm{ph})}$, as shown in equation (6), are the directional transmitting gain and directional receiving gain of the ph-th path between $\mathrm{UE} j$ and AP $k \cdot \chi_{j, k}^{c,(\mathrm{ph})}$ is the amplitude of the ph-th path, and $\delta(\cdot)$ is the Dirac delta function. $\tau_{j, k}^{(\mathrm{ph})}$ is the propagation delay of the ph-th path, which is estimated by the following formula.

$$
\tau_{j, k}^{(\mathrm{ph})}=\frac{d_{j, k}^{(\mathrm{ph})}}{c}
$$

where $d_{j, k}^{(\mathrm{ph})}$ is the distance of the ph-th path between UE $j$ and AP $k$ and $c$ is the speed of light. For analytical tractability, we assume the line-of-sight (LOS) path (i.e., ph $=0$ ) always exists [22] and then the rest of the $\mathrm{PH}$ paths (i.e., from ph $=1$ to $\mathrm{ph}=\mathrm{PH}$ ) are non-line-of-sight (NLOS). As for the LOS path, the amplitude is estimated by the following formula [35].

$$
\chi_{j, k}^{c,(0)}=\frac{\lambda}{4 \pi d_{j, k}^{(0)}}
$$

where $\lambda$ is the wavelength and $\lambda=c / f_{c}$ and $f_{c}$ is the carrier frequency. $d_{j, k}^{(0)}$ is the distance of the LOS path between UE $j$ and AP $k$. As for the NLOS paths, the amplitude is related to both path loss and reflection coefficients, which is estimated by the following formula [35].

$$
\chi_{j, k}^{c,(\mathrm{ph})}=\frac{\lambda}{4 \pi d_{j, k}^{(\mathrm{ph})}} \prod_{\mathrm{rph}=1}^{\mathrm{RPH}} \Gamma_{r \mathrm{ph}}^{(\mathrm{ph})}, \mathrm{ph} \in[1, \mathrm{PH}],
$$

where $\Gamma_{\mathrm{rph}}^{(\mathrm{ph})}$ is the reflection coefficient of the rph-th reflection for the ph-th path and RPH is the number of reflections of the path. Since the reflection loss is very high in the mmWave band [15], we only consider one reflection of a given path (i.e., $\mathrm{RPH}=1$ ). Therefore, formula (10) is simplified as follows.

$$
\chi_{j, k}^{c,(\mathrm{ph})}=\frac{\lambda}{4 \pi d_{j, k}^{(\mathrm{ph})}} \Gamma_{\mathrm{rph}}^{(\mathrm{ph})}, \mathrm{ph} \in[1, \mathrm{PH}] .
$$

Finally, according to [36], the channel gain can be derived by

$$
G_{j, k}^{c,(\mathrm{ph})}=\left|\chi_{j, k}^{c,(\mathrm{ph})} \delta\left(\tau-\tau_{j, k}^{(\mathrm{ph})}\right)\right|^{2} .
$$


Let the directional beam's transmission power from AP $k$ to UE $j$ be $p_{j, k}^{t}$, and then, at the UE $j$ side, the received power from AP $k$ can be determined by

$$
p_{j, k}^{r}=p_{j, k}^{t} \sum_{\mathrm{ph}=0}^{\mathrm{PH}} G_{j, k}^{t,(\mathrm{ph})} G_{j, k}^{r,(\mathrm{ph})} G_{j, k}^{c,(\mathrm{ph})} .
$$

Similarly, the interference power received at UE $j$ from the other APs (e.g., AP $\left.k^{\prime}\left(k^{\prime} \in[1, \cdots, k-1, k+1, \cdots, K]\right)\right)$ and UEs (e.g., UE $j(j \in[1, \cdots, j-1, j+1, \cdots, J]))$ should be

$$
p_{j^{\prime}, k^{k} \rightarrow j, k}^{r}=p_{j^{\prime}, k^{k}}^{t} \sum_{\mathrm{ph}=0}^{\mathrm{PH}} G_{j^{\prime}, k^{\prime} \rightarrow j, k}^{t,(\mathrm{ph})} G_{j^{\prime}, k^{k} \rightarrow j, k}^{r,(\mathrm{ph})} G_{j, k^{k}}^{c,(\mathrm{ph})},
$$

where $G_{j^{\prime}, k^{\prime} \rightarrow j, k}^{t,(\mathrm{ph})}$ and $G_{j^{\prime}, k^{\prime} \rightarrow j, k}^{r,(\mathrm{ph})}$ are the directional transmitting gain and directional receiving gain from the ph-th path between UE $j$ and AP $k$, respectively. According to formula (1), the directional transmit-receive gain of each path can be derived by the following formula.

$$
G_{j^{\prime}, k^{k} \rightarrow j, k}^{t} G_{j^{\prime}, k^{k} \rightarrow j, k}^{r}=\left\{\begin{array}{l}
\frac{2 \pi-\left(2 \pi-\theta_{j^{\prime}, k^{\prime}}^{t}\right) \partial}{\theta_{j, k^{*}}^{t}} \cdot \frac{2 \pi-\left(2 \pi-\theta_{j, k}^{r}\right) \partial}{\theta_{j, k}^{r}}, \text { Condition A, } \\
\frac{2 \pi-\left(2 \pi-\theta_{j, k^{k}}^{t}\right) \partial}{\theta_{j, k^{k}}^{t}} \cdot \partial, \text { Condition B, } \\
\partial \cdot \frac{2 \pi-\left(2 \pi-\theta_{j, k}^{r}\right) \partial}{\theta_{j, k}^{r}}, \text { Condition C, } \\
\partial \cdot \partial, \text { Condition D. }
\end{array}\right.
$$

Let $\phi_{j^{\prime}, k^{k} \rightarrow j, k}^{t}$ and $\phi_{j, k \rightarrow j^{j}, k^{k}}^{r}$ be the beam offset angle from AP $k$ 's (AP $k$ transmits to UE $j$ ) transmitting beam direction to the position of UE $j$ and that from the UE $j$ 's (UE $j$ receives from $\mathrm{AP} k$ ) receiving beam direction to the position of AP $k^{k}$, respectively; Condition A is both $\left|\phi_{j^{\prime}, k^{\prime} \rightarrow j, k}^{t}\right| \leq \theta_{j^{\prime}, k^{\prime}}^{t} / 2$ and $\left|\phi_{j, k \rightarrow j^{\prime}, k^{\prime}}^{r}\right| \leq \theta_{j, k}^{r} / 2$; Condition B is both $\left|\phi_{j^{\prime}, k^{k} \rightarrow j, k}^{t}\right| \leq \theta_{j^{\prime}, k^{\prime}}^{t} / 2$ and $\left|\phi_{j, k \rightarrow j^{\prime}, k^{k}}^{r}\right|>\theta_{j, k}^{r} / 2$; Condition C is both $\left|\phi_{j^{\prime}, k^{\prime} \rightarrow j, k}^{t}\right|>\theta_{j^{\prime}, k^{\prime}}^{t} / 2$ and $\left|\phi_{j, k \rightarrow j^{k}, k^{k}}^{r}\right| \leq \theta_{j, k}^{r} / 2$; Condition D is both $\left|\phi_{j^{\prime}, k^{k} \rightarrow j, k}^{t}\right|>\theta_{j^{\prime}, k^{\prime}}^{t} / 2$ and $\left|\phi_{j, k \rightarrow j^{\prime}, k^{k}}^{r}\right|>\theta_{j, k}^{r} / 2$.

We assume that there is no multiconnectivity capability for UEs, and thus, a UE can only connect to one AP at a time in a dense mmWave cellular network. We set $x_{j, k} \in\{0,1\}$ as the binary association variable. If UE $j \in$ $\mathbb{N}$ is connected to AP $k \in \mathbb{C}$, then $x_{j, k}=1$; otherwise $x_{j, k}$ $=0$. When UE $j$ connects to AP $k$, according to formulas (13) and (14), the signal-to-interference plus noise ratio (SINR) between AP $k$ and UE $j$ is denoted as $\gamma_{j, k}$, which can be derived by

$$
\gamma_{j, k}=\frac{x_{j, k} p_{j, k}^{\mathrm{r}}}{\sum_{j^{\prime} \in \mathbb{N} \backslash j} \sum_{k^{\prime} \in \mathbb{C} \backslash k} x_{j^{\prime}, k} \cdot p_{j^{\prime}, k^{\prime} \rightarrow j, k}^{r}+B \bullet N_{0}},
$$

where $B$ and $N_{0}$ represent the bandwidth of mmWave band and the background noise power spectrum density, respectively. Then, the data rate (throughput) of the link from AP $k$ to UE $j$ is as follows.

$$
r_{j, k}=x_{j, k} B \log _{2}\left(1+\gamma_{j, k}\right) .
$$

The sum data rate of the entire mmWave cellular network (i.e., only the data rate from APs to UEs) is

$$
R=\sum_{j \in \mathbb{N}} \sum_{k \in \mathbb{C}} r_{j, k} .
$$

In order to maximize the sum data rate in (18), the radio access resource management problem for downlink unicast communications can be transformed into the following optimization problem.

$$
\left\{\begin{array}{l}
P 1: \max _{X, P, A} \sum_{j \in \mathbb{N}} \sum_{k \in \mathbb{C}} r_{j, k} \\
\text { s.t. } C_{1}, C_{2}, C_{3}, C_{4}, C_{5}, C_{6} .
\end{array}\right.
$$

In (19), the constraints are specified as follows.

$$
\left\{\begin{array}{l}
C_{1}: x_{j, k} \in\{0,1\}, \forall j \in \mathbb{N}, k \in \mathbb{C}, \\
C_{2}: \sum_{k \in \mathbb{C}} x_{j, k} \leq 1, \forall j \in \mathbb{N}, \\
C_{3}: \sum_{j \in \mathbb{N}} x_{j, k} \leq n_{\text {beam }}, \forall k \in \mathbb{C}, \\
C_{4}: \sum_{j \in \mathbb{N}} x_{j, k} p_{j, k}^{t} \leq p_{k}^{\max }, \forall k \in \mathbb{C}, \\
C_{5}: \theta_{\min } \leq \theta_{j, k}^{t}, \theta_{j, k}^{r} \leq \theta_{\max }, \forall j \in \mathbb{N}, k \in \mathbb{C}, \\
C_{6}: \sum_{j \in \mathbb{N}} x_{j, k} \theta_{j, k}^{t}, \sum_{j \in \mathbb{N}} x_{j, k} \theta_{j, k}^{r} \leq 2 \pi, k \in \mathbb{C},
\end{array}\right.
$$

where $X$ is a matrix with element $x_{j, k}, P$ is a matrix with element $p_{j, k}^{t}$, and $A$ is a matrix with element $\theta_{j, k}^{t}$ or $\theta_{j, k}^{r}$. Constraint $C_{2}$ indicates that each UE can only connect to one AP. Constraint $C_{3}$ ensures that the number of UEs that an AP can serve is not more than $n_{\text {beam }}$. Constraint $C_{4}$ means that the power consumption constraint for each AP cannot exceed $p_{k}^{\max }$. Constraint $C_{5}$ indicates that the beam width is limited to $\left[\theta_{\min }, \theta_{\max }\right]$. Constraint $C_{6}$ stipulates that for an AP, the sum of beam widths used for serving the connected UEs cannot exceed $2 \pi$, since the beams of a node are assumed to be nonoverlapping in Section 3.1. However, the optimization problem $P 1$ could not be solved directly because it is NP-hard and not convex. Accordingly, we design an algorithm to find a suboptimal solution for problem $P 1$ in Section 5 . 


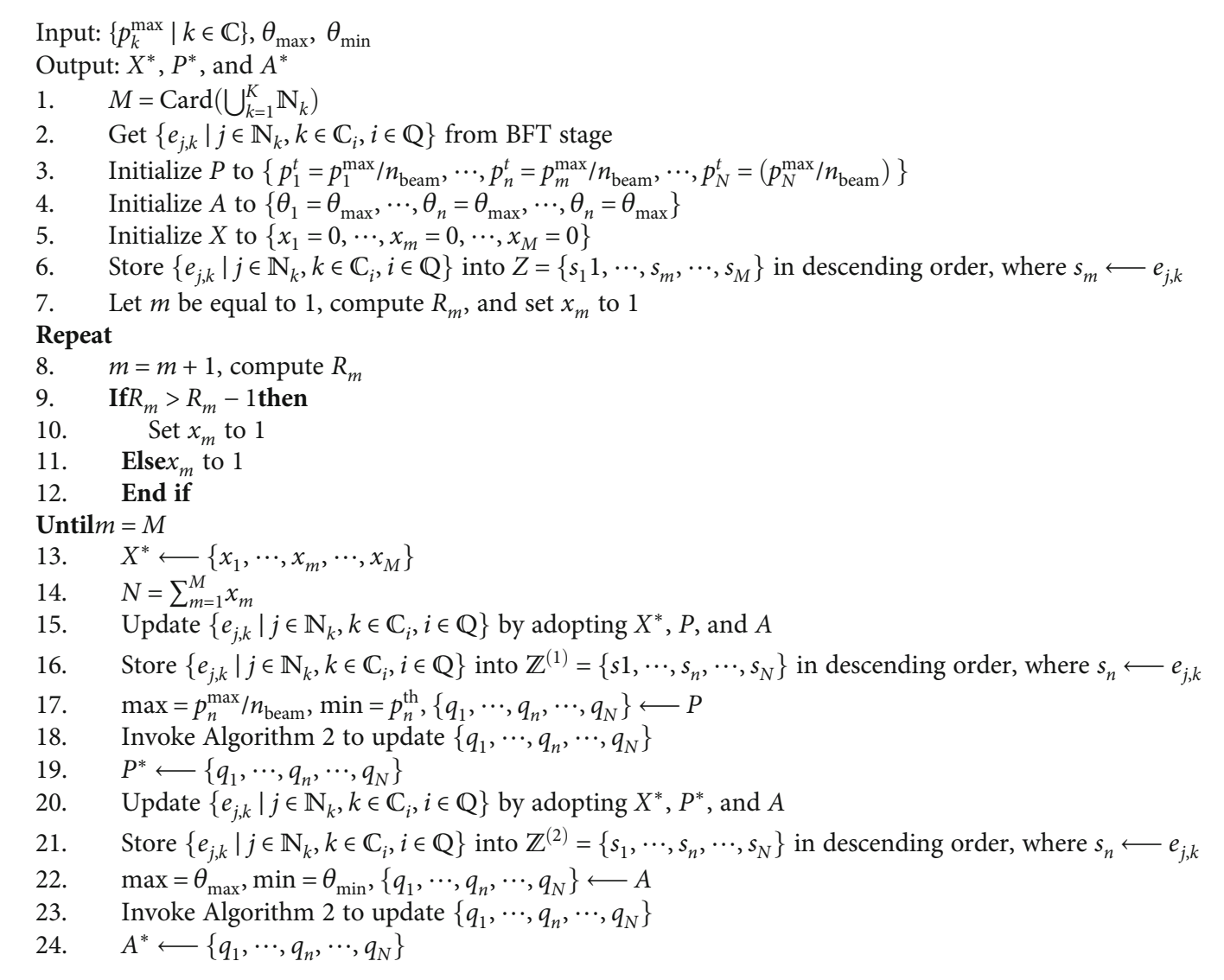

Algorithm 1: BFT information-aided radio access resource management algorithm for solving problem $P 2$.

\section{Radio Access Resource Management in Dense mmWave Cellular Network}

In this section, we propose a hierarchical BFT informationaided radio access resource management algorithm to solve problem $P 1$ so as to reduce the interference and improve the energy efficiency of dense mmWave cellular network.

Since the path loss of mmWave band is serious and the transmission power is limited in practical scenarios, a UE may not pick up signals from all the APs in the network. That is to say, a UE may only connect to its available surrounding APs. Each AP may only get a set of candidate UEs based on the hierarchical BFT results in Section 3.2. We judge whether there is a direct communication link between a UE (e.g., $j$ ) and an $\mathrm{AP}$ (e.g., $k$ ) by observing the receiving bit error rate (BER).

According to the formula of estimating the bit transmission success rate in [37] (i.e., $f\left(\gamma_{j}\right)=1-e^{-0.5 \gamma_{j}}$; here, $f\left(\gamma_{j}\right)$ is UE $j$ 's bit transmission success rate, and $\gamma_{j}$ is the corresponding SINR value), we know that the corresponding BER value can be estimated by $e^{-0.5 \gamma_{j}}$. When the acceptable BER in this paper is determined and denoted as $\mathrm{BER}_{j, k}^{\mathrm{th}}$, which is the BER threshold of UE $j$ 's receiving data from AP $k$, the corresponding SINR threshold (i.e., $\gamma_{j, k}^{\mathrm{th}}$ ) can be estimated by

$$
\gamma_{j, k}^{\mathrm{th}}=-2 \ln \mathrm{BER}_{j, k}^{\mathrm{th}} .
$$

In order to ensure that the BER level of $\mathrm{UE} j$ 's receiving data from AP $k$ is not higher than $\mathrm{BER}_{j, k}^{\mathrm{th}}$, the transmission power of AP $k$ should not be lower than the power threshold $p_{j, k}^{\text {th }}$, which is estimated as follows.

$$
p_{j, k}^{\text {th }}=\frac{\gamma_{j, k}^{\text {th }} \bullet B \cdot N_{0}}{\sum_{\mathrm{ph}=0}^{\mathrm{PH}} G_{j, k}^{t,(\mathrm{ph})} G_{j, k}^{r,(\mathrm{ph})} G_{j, k}^{c,(\mathrm{ph})}} .
$$

If $\gamma_{j, k}$ is not less than $\gamma_{j, k}^{\mathrm{th}}$, we believe that there is a direct communication link to UE $j$ from AP $k$. For a set of candidate UEs of AP $k(k \in \mathbb{C})$, the directional link's quality (e.g., $\left.\gamma_{j, k}\right)$ between each UE $j(j \in \mathbb{N})$ and AP $k$ should be greater than a predefined threshold (e.g., $\gamma_{j, k}^{\text {th }}$ ), where the set of candidate UEs of AP $k$ is denoted as $\mathbb{N}_{k}$. Therefore, expressions (19) and (20) can be rewritten as

$$
\left\{\begin{array}{l}
P 2: \max _{X, P, A} \sum_{k \in \mathbb{C}} \sum_{j \in \mathbb{N}_{k}} r_{j, k} \\
\text { s.t. } C_{1}, C_{2}, C_{3}, C_{4}, C_{5}, C_{6} .
\end{array}\right.
$$




$$
\left\{\begin{array}{l}
C_{1}: x_{j, k} \in\{0,1\}, \forall j \in \mathbb{N}_{k}, k \in \mathbb{C}, \\
C_{2}: \sum_{k \in \mathbb{C}} x_{j, k} \leq 1, \forall j \in \mathbb{N}_{k}, \\
C_{3}: \sum_{j \in \mathbb{N}_{k}} x_{j, k} \leq n_{\text {beam }}, \forall k \in \mathbb{C}, \\
C_{4}: \sum_{j \in \mathbb{N}_{k}} x_{j, k} p_{j, k}^{t} \leq p_{k}^{\max }, \forall k \in \mathbb{C}, \\
C_{5}: \theta_{\min } \leq \theta_{j, k}^{t}, \theta_{j, k}^{r} \leq \theta_{\text {max }}, \forall j \in \mathbb{N}_{k}, k \in \mathbb{C}, \\
C_{6}: \sum_{j \in \mathbb{N}_{k}} x_{j, k} \theta_{j, k}^{t}, \sum_{j \in \mathbb{N}_{k}} x_{j, k} \theta_{j, k}^{r} \leq 2 \pi, k \in \mathbb{C} .
\end{array}\right.
$$

In the proposed BFT, when a UE concurrently transmits its training response beacon frames in all its beams, its surrounding APs that can receive these frames will firstly transmit their built beacon frames for announcing training results to their associated SBSs. If these SBSs independently perform the merge operation of training results and then feedback the results to the corresponding APs, some of these APs will reply to this UE with a response packet, respectively. That is to say, this UE may receive the multiple APs' response packets, and thus, it should decide which AP to associate. Since these SBSs are adjacent to each other and also their numbers are usually small, we believe that they can exchange merge operation results with each other through the dedicated channels at a small cost, which can ensure that only the AP that has the best channel quality between itself and this UE will transmit one response packet to this UE.

For each SBS (e.g., $i$ ), it can obtain all the SNR values (i.e., $\left.\left\{\gamma_{j, k} \mid j \in \mathbb{N}_{k}, k \in \mathbb{C}_{i}, i \in \mathbb{Q}\right\}\right)$ in its coverage when the proposed BFT is done. However, using SNR directly to measure channel quality is not always an effective method. Therefore, considering the data rate per unit of power consumption (DRPC) in a link to measure channel quality can better reflect the goal of high energy efficiency pursued by the next-generation wireless network, which is formulated as follows.

$$
e_{j, k}=\frac{B \log _{2}\left(1+\gamma_{j, k}\right)}{p_{j, k}^{t}+P_{\mathrm{RF}}} .
$$

In (25), $e_{j, k}$ is the energy efficiency of the link from AP $k$ to $\mathrm{UE} j$, and $P_{\mathrm{RF}}$ is the power consumed by a radio frequency (RF) chain, where $P_{\mathrm{RF}}$ is set to $0.0344 \mathrm{~W}$ in [38].

For the sake of simplicity of analysis, we store all the DRPC values into $\mathbb{Z}=\left\{s_{1}, \cdots, s_{m}, \cdots, s_{M}\right\}$ in descending order. Then, there is a one-to-one match between the elements of $\mathbb{Z}$ and those of $\left\{e_{j, k} \mid j \in \mathbb{N}_{k}, k \in \mathbb{C}_{i}, i \in \mathbb{Q}\right\}$, where $M=\operatorname{Card}\left(\bigcup_{k=1}^{K} \mathbb{N}_{k}\right)$ and $\operatorname{Card}(\bullet)$ stands for the number of elements in a set and $\bigcup_{k=1}^{K} \mathbb{N}_{k}$ represents the set of all the UEs associated by all the APs. In addition, the number of elements in $X, P$, and $A$ is also $M$.

The problem described in (23) and (24) is also NP-hard and not convex, so it cannot be solved directly. The proposed algorithm decomposes this problem into three subproblems,

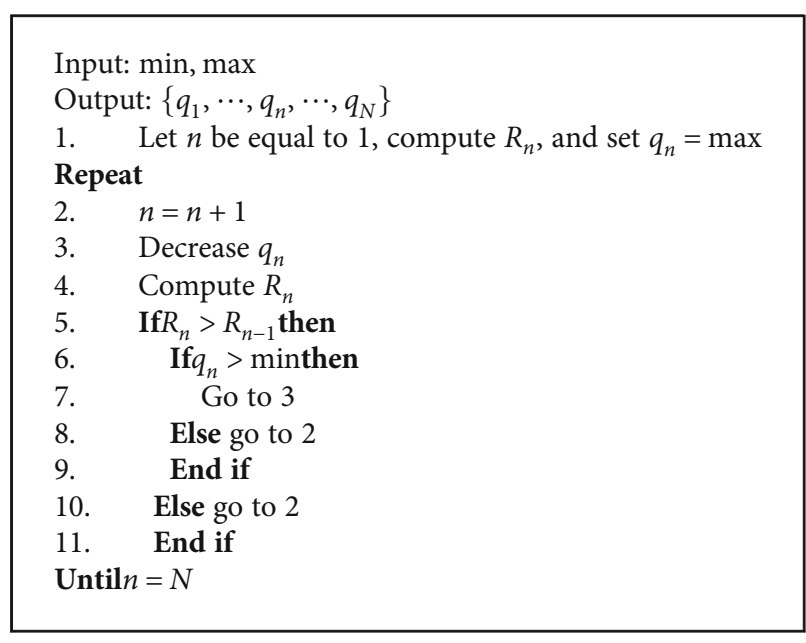

Algorithm 2: The decreasing-search-one-by-one.

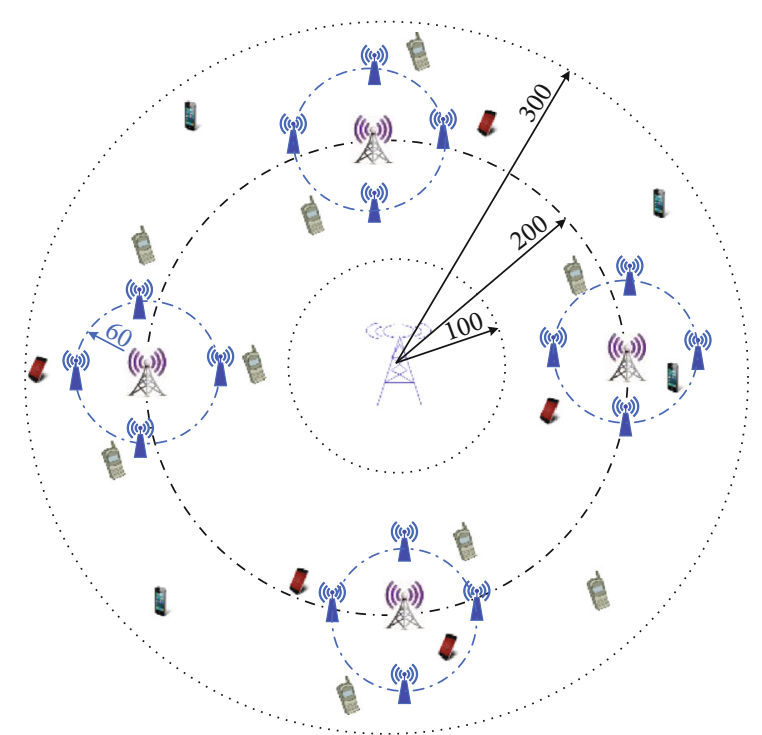

FIGURE 4: mmWave cellular network topology used for simulation.

TABLE 1: The node configuration of simulation scenarios.

\begin{tabular}{lcccc}
\hline $\begin{array}{l}\text { Scenario } \\
\text { name }\end{array}$ & $\begin{array}{c}\text { Number of } \\
\text { MBSs }\end{array}$ & $\begin{array}{c}\text { Number of } \\
\text { SBSs }\end{array}$ & $\begin{array}{c}\text { Number of } \\
\text { APs }\end{array}$ & $\begin{array}{c}\text { Number of } \\
\text { UEs }\end{array}$ \\
\hline Scenario 1 & 1 & 4 & 16 & 32 \\
Scenario 2 & 1 & 4 & 32 & 64 \\
Scenario 3 & 1 & 8 & 32 & 64 \\
Scenario 4 & 1 & 8 & 64 & 128 \\
\hline
\end{tabular}

which can find the suboptimal solution. Firstly, we initialize $P$ and $A$ to obtain the optimized $X^{*}$. Then, we use the optimized $X^{*}$ and initialized $A$ to get the optimized $P^{*}$. Finally, we adopt the optimized $X^{*}$ and $P^{*}$ to find the optimized $A^{*}$.

In the proposed algorithm description shown in Algorithm 1 , to obtain the optimized $X^{*}, P$ and $A$ are firstly initialized to the average power (i.e., $p_{k \text {,ini }}^{t}=p_{k}^{\max } / n_{\text {beam }}$ ) and the maximum beam width (i.e., $\theta_{j, k}^{t}, \theta_{j, k}^{r}=\theta_{\max }$ ), respectively. After that, all the elements in $X$ are initialized to 0 . Then, the 
TABLE 2: The simulation parameters.

\begin{tabular}{lcc}
\hline Symbol & Description & Value \\
\hline$p_{j}^{\max }$ & Maximum transmission power for UE & $23 \mathrm{dBm}$ \\
$p_{k}^{\max }$ & Maximum transmission power for AP & $30 \mathrm{dBm}$ \\
$p_{i}^{\max }$ & Maximum transmission power for SBS and MBS & $43 \mathrm{dBm}$ \\
$f_{c}$ & Carrier frequency & $60 \mathrm{GHz}$ \\
$B$ & Bandwidth & $1 \mathrm{GHz}$ \\
$\mathrm{PH}$ & Maximum number of paths \\
$\Gamma$ & Reflection coefficient & 3 \\
$\theta_{\min }$ & Minimum beam width \\
$\theta_{\max }$ & Maximum beam width \\
$\partial$ & The side lobe gain & 0.3 \\
$n_{\text {beam }}$ & The number of beams for each node & $10^{\circ}$ \\
$N_{0}$ & Channel noise power spectrum density & $30^{\circ}$ \\
\hline
\end{tabular}

algorithm selects the AP and UE pair corresponding to $s_{1}$ and then calculates the sum data rate $R_{1}$ according to $\sum_{k \in \mathbb{C}}$ $\sum_{j \in \mathbb{N}_{k}} r_{j, k}$ and sets $x_{1}$ to 1 . Next, the algorithm selects the AP and UE pair corresponding to $s_{2}$ and calculates the sum data rate $R_{2}$. If $R_{2}>R_{1}$, the algorithm sets $x_{2}$ to 1 ; otherwise, it sets $x_{2}$ to 0 .

By repeating the above steps, the algorithm selects the AP and UE pair corresponding to $s_{m}$ and calculates the sum data rate $R_{m}$. If $R_{m}>R_{m}-1, x_{m}$ is set to 1 ; otherwise, $x_{m}$ is set to 0 , until all the $x_{m}$ are set. Therefore, the algorithm can get the optimized $X *$ after mapping $x_{m}$ to $x_{j, k}$. Since $X^{*}$ indicates which AP each UE should connect to, the beam directions are determined.

After this stage, the algorithm can use the optimized $X^{*}$ and the maximum beam width vector $A$ to obtain power allocation vector $P^{*}$. The algorithm starts from the directional link with the highest DRPC value determined by $X^{*}, P$, and $A$. Then, it decreases the transmission power $p_{m}^{t}$ from the average power $p_{m}^{a}$ in $P$. If the sum data rate increases, the algorithm continues to reduce the transmission power $p_{m}^{t}$ until it reaches $p_{m}^{\text {th }}$; otherwise, it optimizes the transmission power of the link with the second highest DRPC value. Repeating the above steps until all the $p_{m}^{t}$ are optimized, we can get $P^{*}$ after mapping $p_{m}^{t}$ to $p_{j, k}^{t}$.

Finally, the optimized $A^{*}$ can be obtained by applying $X^{*}$ and $P^{*}$ into the process similar to the one above. That is to say, the algorithm starts from the directional link with the highest DRPC value determined by $X^{*}, P^{*}$, and $A$. Then, it decreases beam width from $\theta_{\max }$. If the sum data rate increases, the algorithm continues to reduce beam width until it reaches $\theta_{\text {min }}$; otherwise, it optimizes the beam width of the link with the second highest DRPC value. Repeating the above steps until all the $\theta_{m}$ are optimized, we can get $A^{*}$ after mapping $\theta_{m}$ to $\theta_{j, k}^{t}$ or $\theta_{j, k}^{r}$. Therefore, $X^{*}, P^{*}$, and $A^{*}$ can be obtained.

It is noteworthy that $M$ is greater than $N$ in Algorithm 1, which means that the number of elements in $P^{*}$ is less than that of $X^{*}$. When $x_{j, k}$ is equal to $0, P^{*}$ is filled with 0 to make the number of elements in $P^{*}$ equal to that of $X^{*}$. Similarly, the number of elements in $A^{*}$ should be equal to that of $X^{*}$ When $x_{j, k}$ is equal to $0, A^{*}$ is also filled with 0 to reach the same number of elements (i.e., $M$ ).

The above algorithm invokes the following algorithm based on the idea of decreasing search one by one.

\section{Performance Evaluation}

6.1. Simulation Parameter Settings for Radio Resource Management Scheme. As shown in Figure 4, we consider a circular plane simulation area with the radius of $300 \mathrm{~m}$, in which the MBS is located. Meanwhile, the SBSs are uniformly deployed in a circular ring with the radius of $200 \mathrm{~m}$ centered at the MBS, while the APs are uniformly deployed in a set of circular rings with the radius of $60 \mathrm{~m}$ centered at each SBS. Such setting is only to facilitate the construction of the simulation environment, and it does not affect the analysis and verification of the scheme feasibility.

In addition, we also consider the four scenarios shown in Table 1, where all the UEs are randomly distributed in the ring-shaped area with the radius ranging from $100 \mathrm{~m}$ to $300 \mathrm{~m}$ centered on the MBS. In Scenario 1, there are one MBS and four SBSs, where there are four APs and eight UEs in each SBS, respectively. In Scenario 2, there are one MBS and four SBSs, where eight APs and sixteen UEs are deployed in each SBS, respectively. In Scenario 3, there are one MBS and eight SBSs, where there are four APs and eight UEs in each SBS, respectively. In Scenario 4, there are one MBS and eight SBSs, where eight APs and sixteen UEs are deployed in each SBS, respectively.

The work in [19] is most similar to ours. When it is applied to the scenario in Figure 4, only two steps for the BFT process are required, as mentioned in Section 3.3. For simplicity, we call it the comparison scheme. We adopt the total number of training frames to measure the overhead of BFT for the proposed scheme and the comparison scheme. 


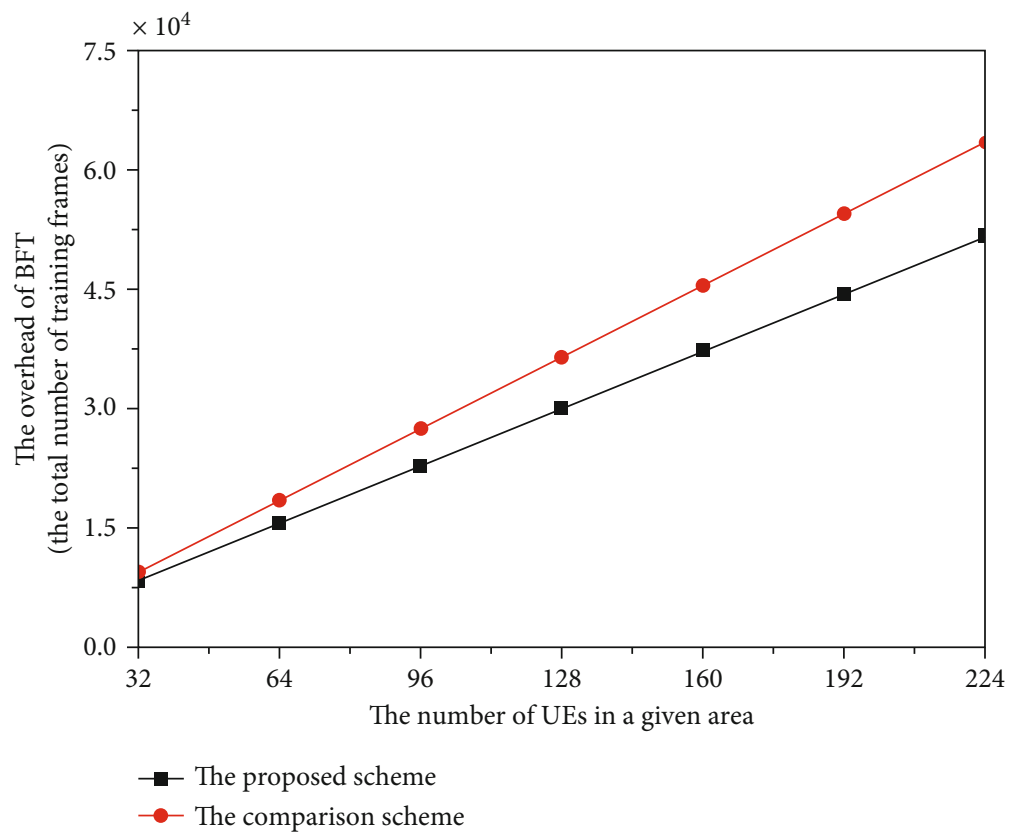

(a)

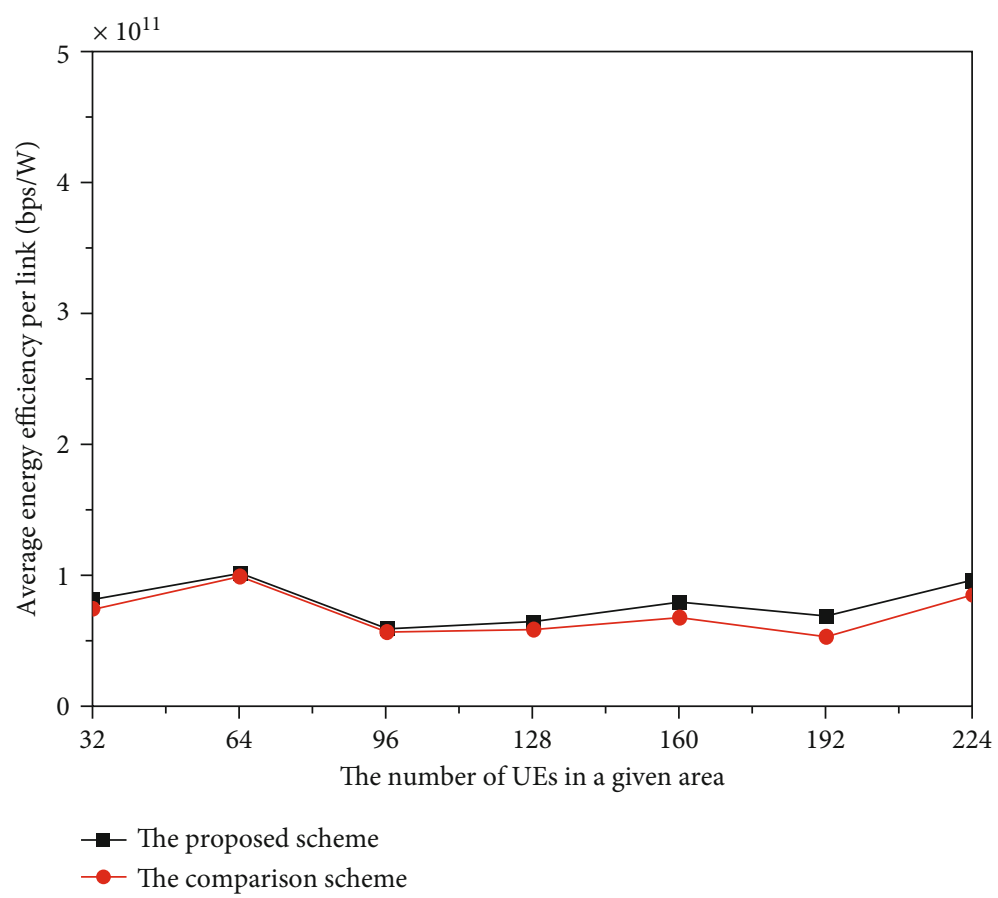

(b)

Figure 5: Continued. 


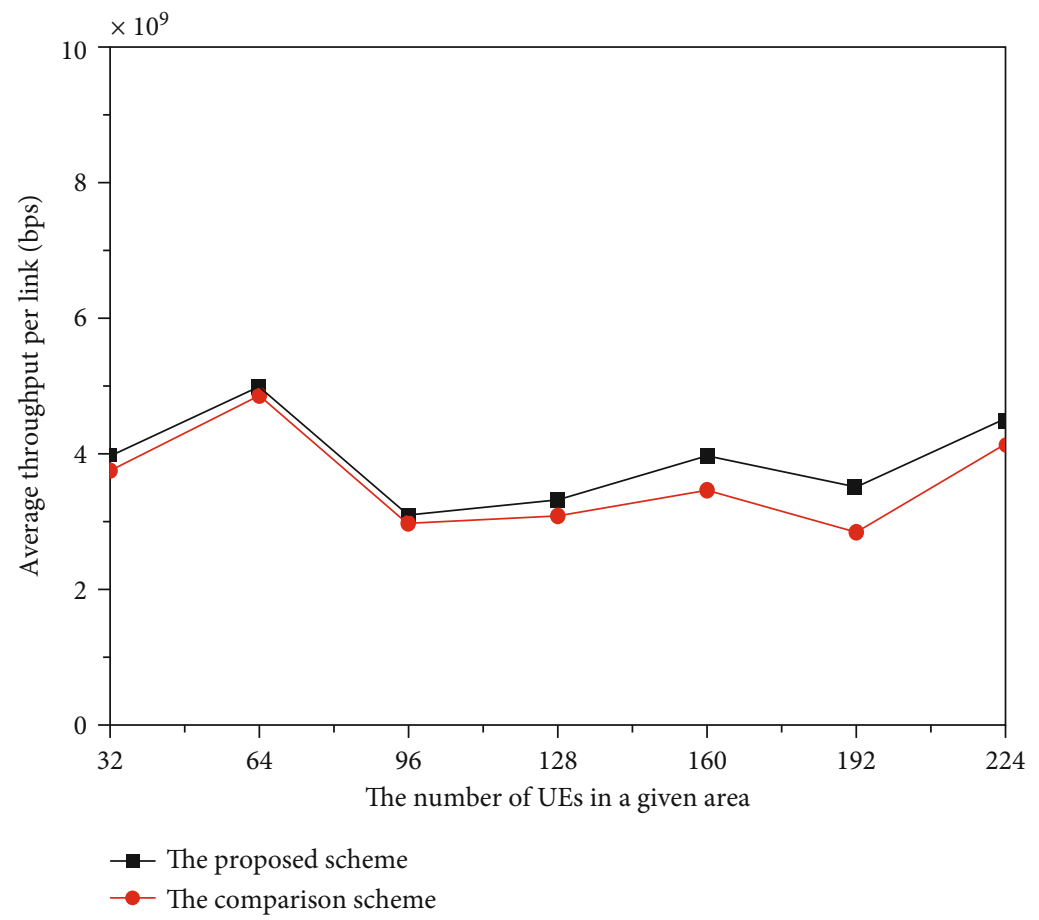

(c)

FIGURE 5: The performance variation of the two schemes with the number of UEs in a given area. (a) The overhead of BFT; (b) average energy efficiency per link; (c) average throughput per link.

Unless otherwise stated, the simulation parameters are listed in Table 2.

6.2. Simulation Results and Analysis for Radio Resource Management Scheme. We conduct a total of five groups of simulation experiments. In the first group of simulation experiments, we set the number of SBSs to 4 and the number of APs to 16 . Also, we set the decreasing step size for power and beam width to one percent of their maximum values, respectively. Based on these parameter settings, we compare the performance of the proposed scheme to that of the comparison scheme by changing the number of UEs in a given area. From Figure 5(a), we can see that the overhead of BFT increases with the number of UEs in a given area. The main reason is that more UEs require more training frames. Also, we observe that the proposed scheme outperforms the comparison scheme in terms of the overhead of BFT, which is consistent with the analysis result in Section 3.3. From Figures 5(b) and 5(c), we can see that the change in the number of UEs has a slight effect on the average energy efficiency and throughput per link. Moreover, this effect is random. This is because the difference in the number and location distribution of UEs leads to the random variation of mutual interference. Also, we observe that the proposed scheme outperforms the comparison scheme in terms of the average energy efficiency and throughput per link. This phenomenon is mainly attributed to the fact that the proposed scheme prioritizes radio access link selection based on link energy efficiency.

In the second group of simulation experiments, we set the number of SBSs to 4 , the number of APs to 16 , and the num- ber of UEs in a given area to 224. Based on these parameter settings, we compare the performance of the proposed scheme to that of the comparison scheme by changing the decreasing step size for power and beam width. From Figure 6(a), we can see that the overhead of BFT is not affected by the change of step size. The reason is that the number of UEs is fixed, and thus, the total number of training frames is almost unchanged. From Figures 6(b) and 6(c), we can see that the change of step size has almost no influence on the average energy efficiency and throughput per link.

If the step size is further reduced to less than $1 \%$, the performance of the convergence point may be better or remain unchanged. However, it is bound to take a longer time to reach the convergence point due to the larger search space. On the contrary, if the step size is too large, the convergence point of the algorithm may be significantly different from the optimal solution. The step size adopted by our simulation is still relatively small when the number of UEs in a given area is set to 224. Therefore, the convergence speed can still be accelerated by increasing the step size in such a case.

In the third group of simulation experiments, we set the number of SBSs to 4 and the number of APs to 16. Also, the number of UEs in a given area is set to 224, while the decreasing step size for power and beam width is set to one percent of their maximum values, respectively. Based on these parameter settings, we compare the performance of the proposed scheme to that of the comparison scheme under the different ambient noise power density values. From Figure $7(\mathrm{a})$, we can see that the overhead of BFT is not affected by the change of ambient noise. The corresponding 


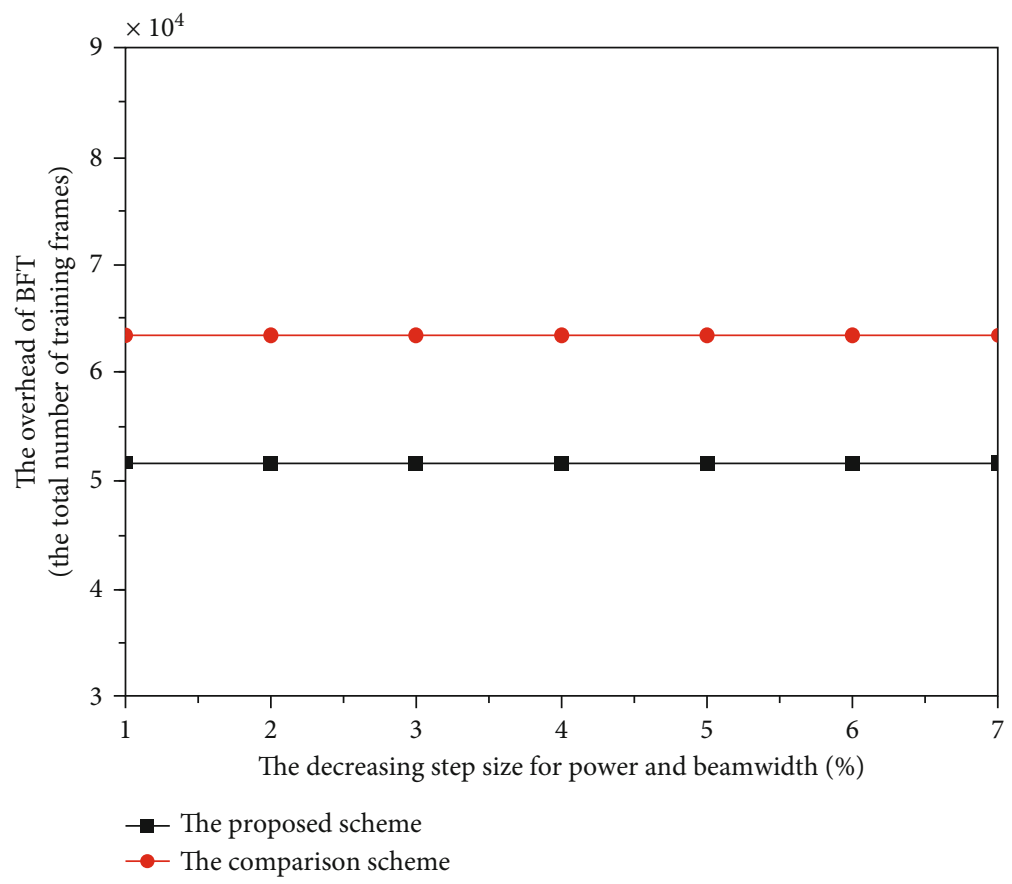

(a)

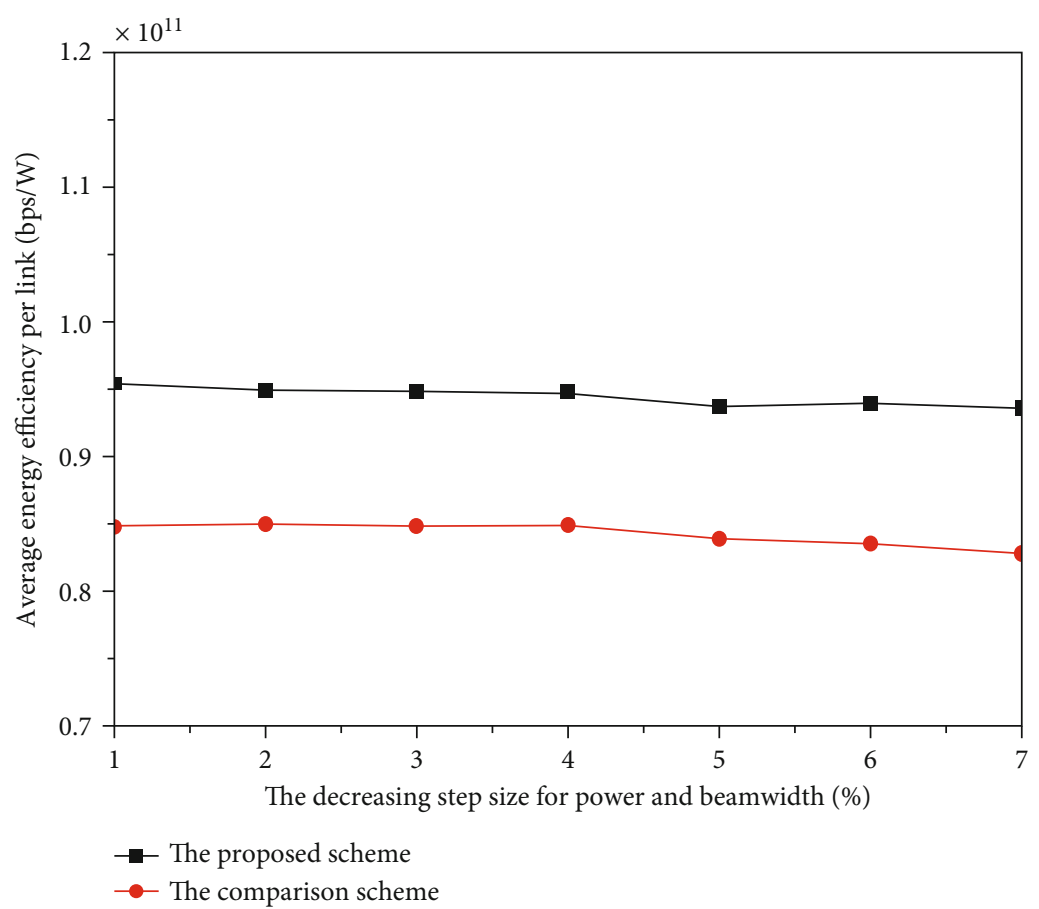

(b)

Figure 6: Continued. 


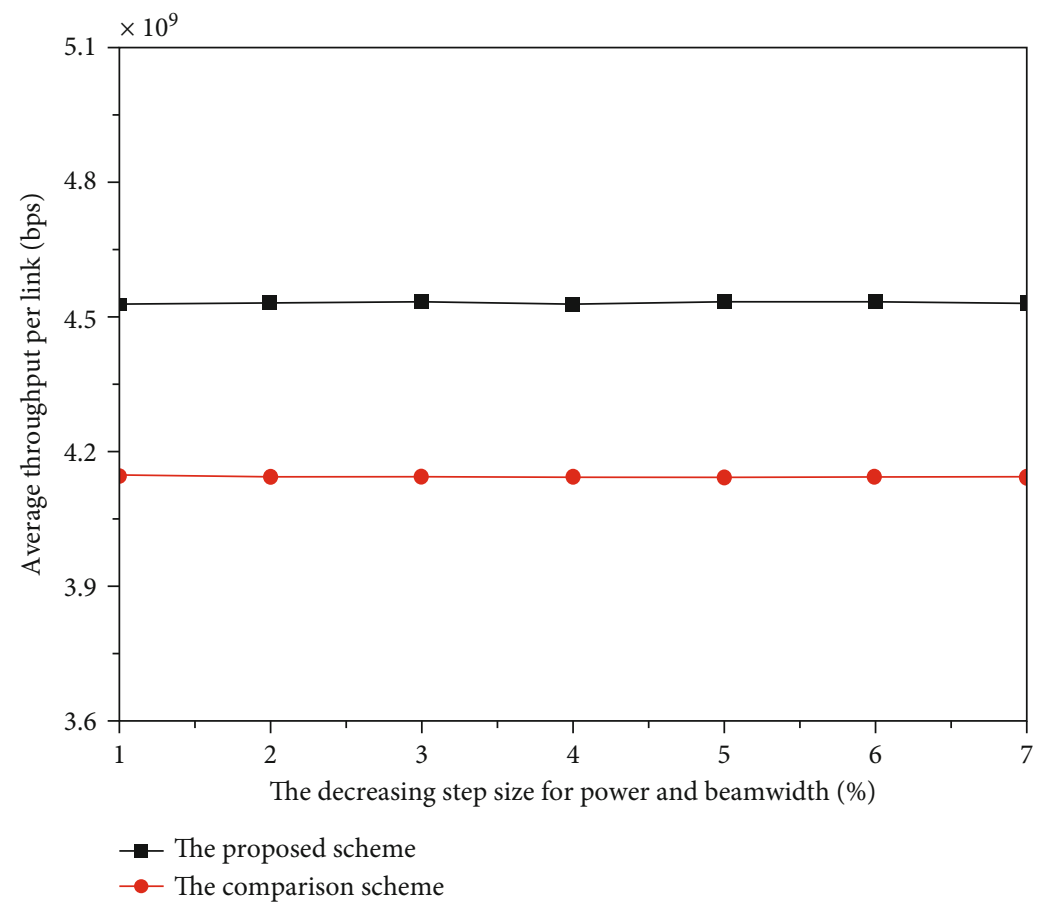

(c)

FIGURE 6: The performance variation of the two schemes with the decreasing step size for power and beam width. (a) The overhead of BFT; (b) average energy efficiency per link; (c) average throughput per link.

explanation is the same as that in Figure 6(a). From Figures $7(\mathrm{~b})$ and $7(\mathrm{c})$, we can see that the change of ambient noise has almost no influence on the average energy efficiency and throughput per link when ambient noise is greater than a certain threshold. However, when ambient noise is very small, the average energy efficiency and throughput per link are greatly improved. The reason is that, when the transmission power is fixed, very low noise will produce very high SNR, and thus, there is a significant throughput improvement according to the Shannon theorem. However, when ambient noise is greater than a certain threshold, although the SNR still increases with the decrease of ambient noise, this change has little impact on throughput according to the Shannon theorem.

In the fourth group of simulation experiments, by changing the decreasing step size for power and beam width, we show the performance of the proposed scheme under the four scenarios. From Figure 8(a), we can see that, among the four scenarios, the overhead of BFT in Scenario 1 is the smallest while that in Scenario 4 is the largest. This is because Scenario 4 has the most UEs and APs and Scenario 1 has the least UEs and APs in all the four scenarios. In addition, Scenario 2 has the same number of UEs and APs as that of Scenario 3, but it has less SBSs than Scenario 3. Although fewer SBSs can help save some training frames, the merging load of training frames is borne by fewer SBSs, and thus, the total overhead of BFT increases slightly. From Figures 8(b) and 8 (c), we also observe that, among the four scenarios, Scenario 1 shows the best average energy efficiency and throughput per link, Scenario 2 and Scenario 3 follow it, and Scenario 4 shows the worst average energy efficiency and throughput per link. The reason behind this phenomenon can be found from the difference in the density of nodes (i.e., UEs, APs, and SBSs), where the lower the density of nodes leads to the less interference between nodes.

In addition, from Figures 8 (b) and 8(c), we see that the curve of Scenario 3 has a turning point when the decreasing step size is $6 \%$. This is because, when the decreasing step size is larger, the probability of poor performance at the convergence point increases. But that does not mean it is necessarily going to get worse. Under Scenario 3 with the step size $6 \%$, this case occurs, which is a manifestation of randomness caused by the random distribution of UEs.

In the fifth group of simulation experiments, we set the decreasing step size for power and beam width to one percent of their maximum values, respectively, and compare the performance of the proposed scheme under the four scenarios by changing the different ambient noise power density values. From Figure 9(a), we can see that the four scenarios show the similar performance to that in Figure 8(a) in terms of the overhead of BFT. Moreover, the interpretation of Figure 8(a) also applies to Figure 9(a). From Figures 9(b) and $9(\mathrm{c})$, we can see that, under the four scenarios, the proposed scheme shows the similar performance variation trend to those in Figures 7(b) and 7(c), respectively. Moreover, we can also observe that the performance differences in the four scenarios are similar to those in Figures 8(b) and 8(c), respectively, especially in the case of the ambient noise greater than a certain threshold. Therefore, the corresponding explanations apply here. 


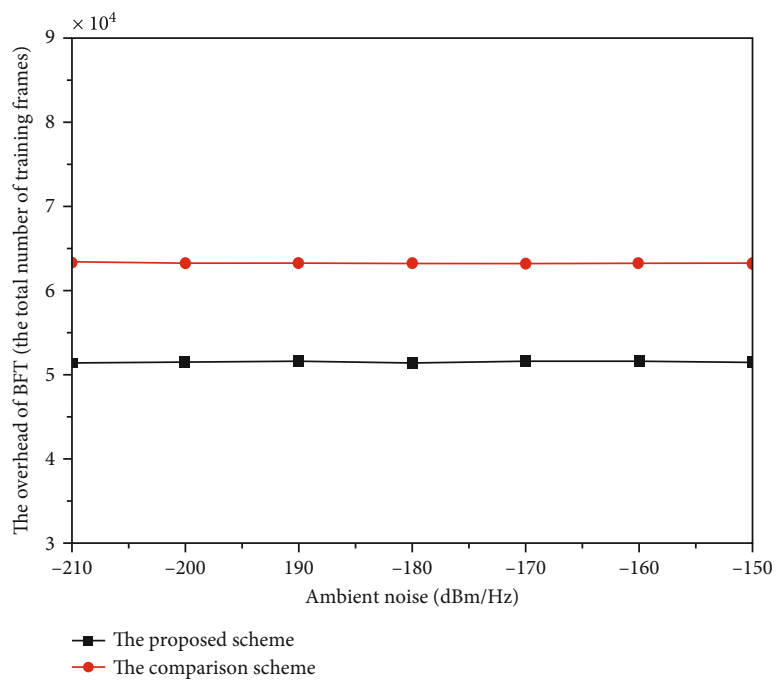

(a)

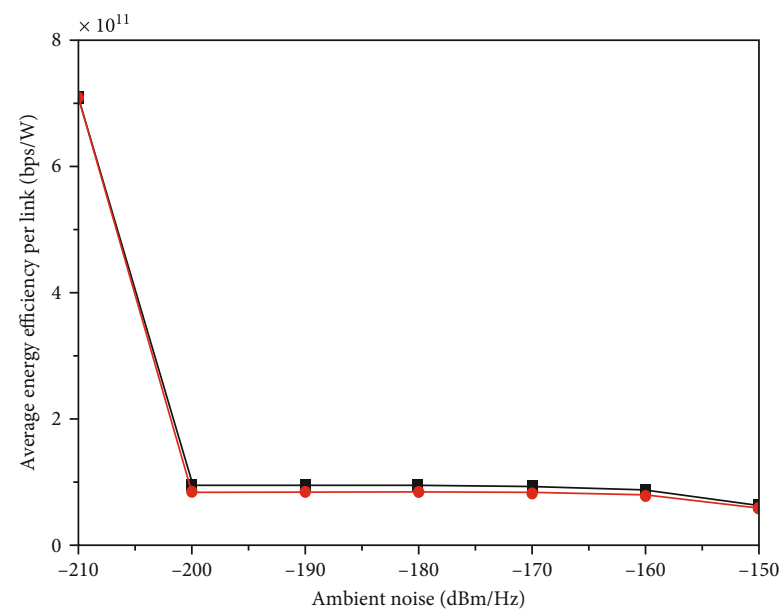

- The proposed scheme

$\rightarrow$ The comparison scheme

(b)

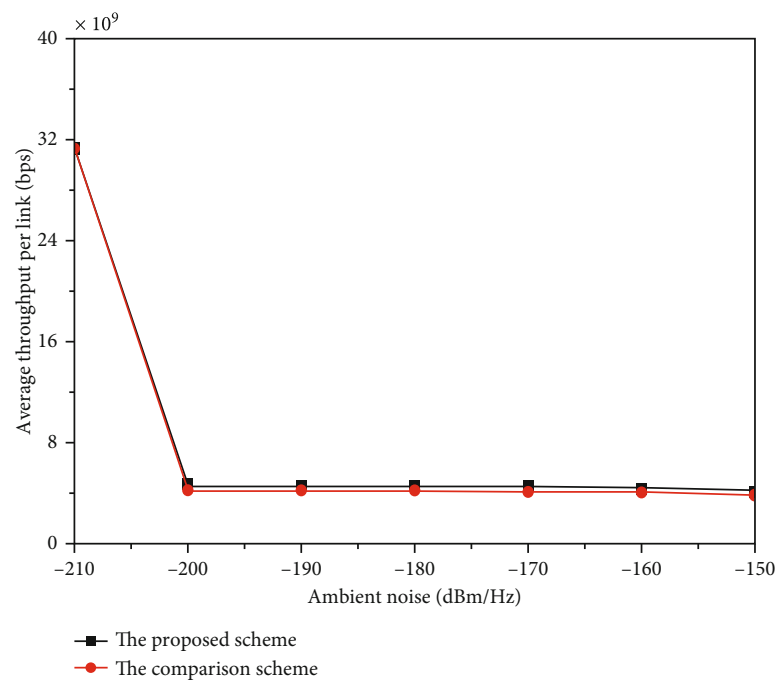

(c)

FIGURE 7: The performance variation of the two schemes with ambient noise. (a) The overhead of BFT; (b) average energy efficiency per link; (c) average throughput per link. 


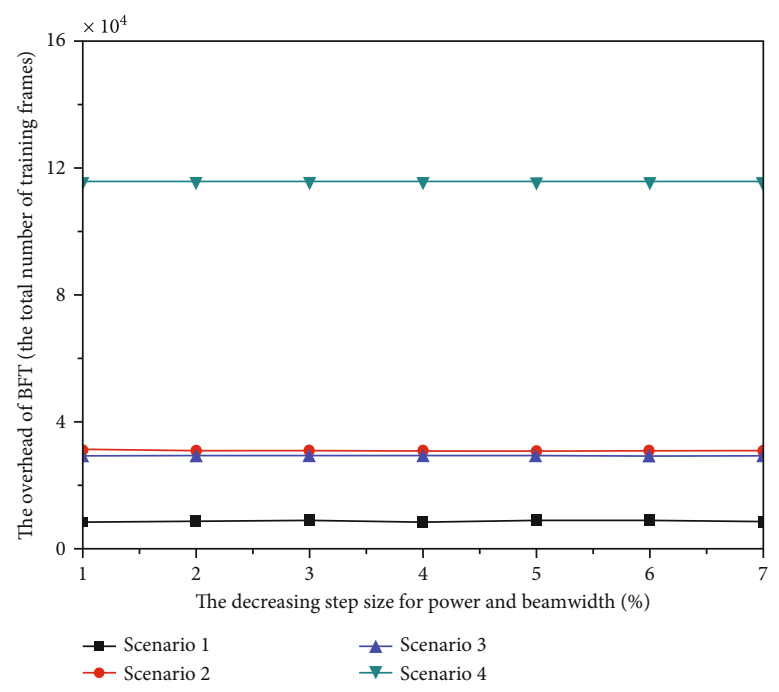

(a)

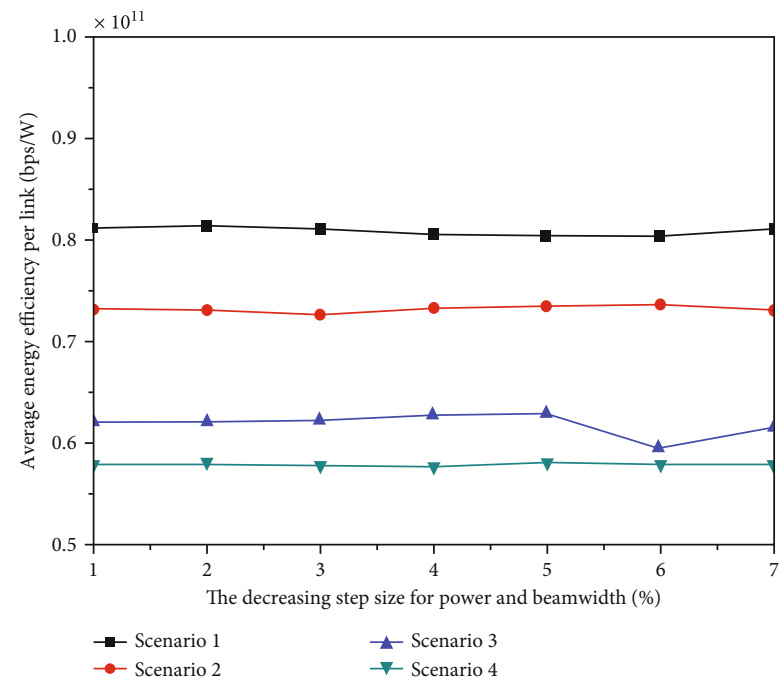

(b)

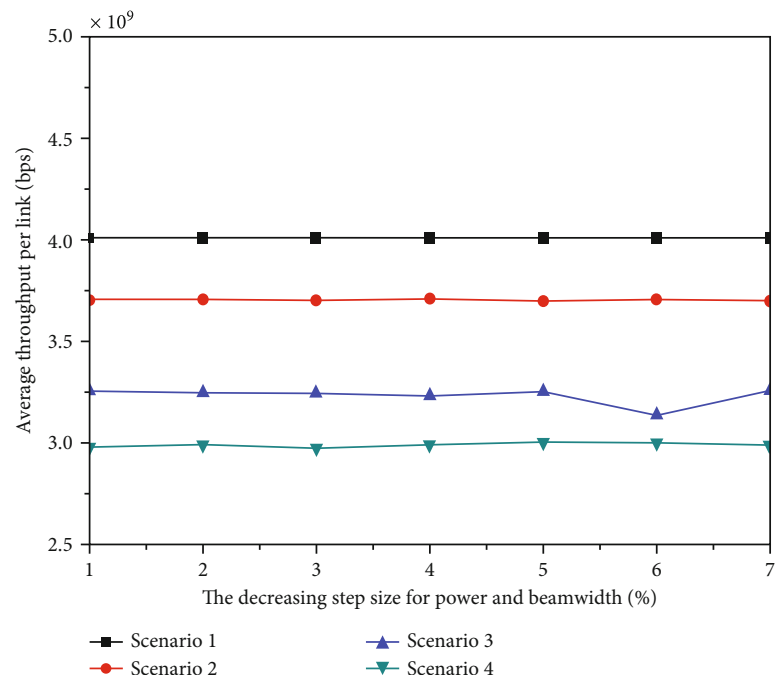

(c)

FIGURE 8: The performance variation of the proposed scheme with the decreasing step size for power and beam width under four scenarios. (a) The overhead of BFT; (b) average energy efficiency per link; (c) average throughput per link. 


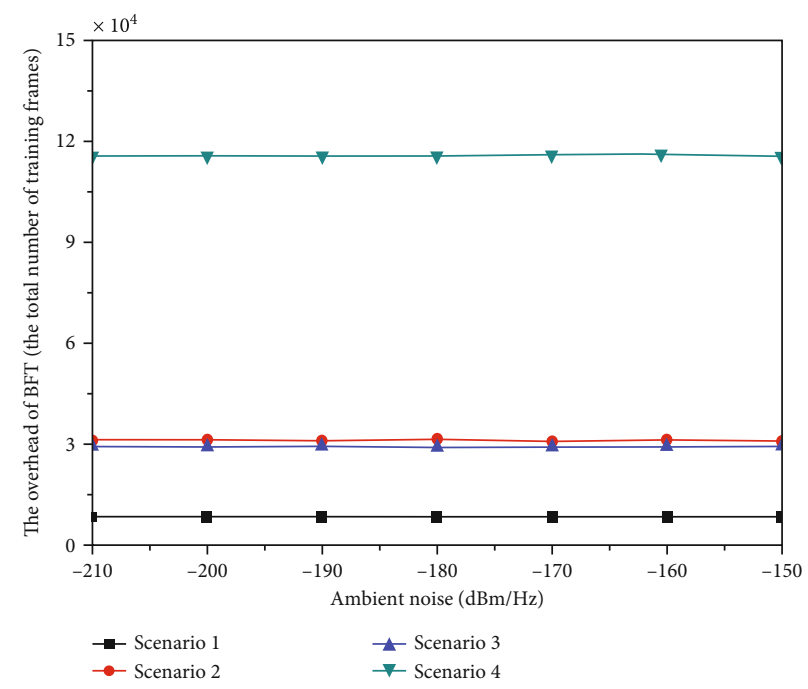

(a)

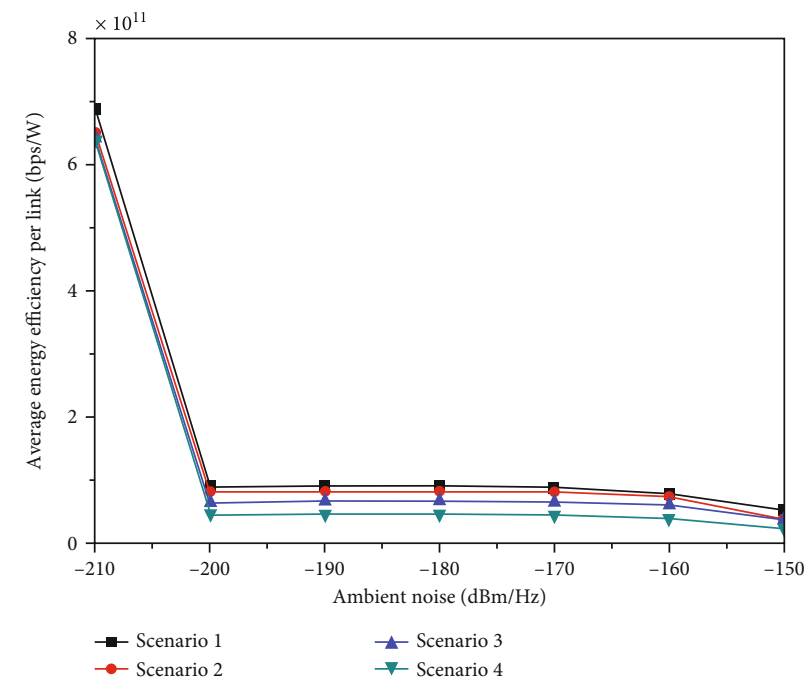

(b)

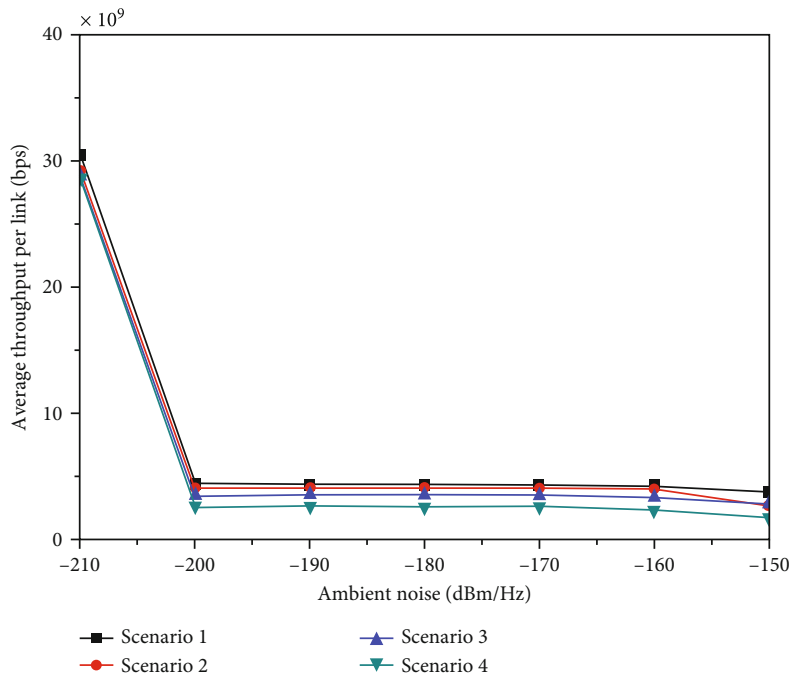

(c)

FIGURE 9: The performance variation of the proposed scheme with ambient noise under four scenarios. (a) The overhead of BFT; (b) average energy efficiency per link; (c) average throughput per link. 


\section{Conclusion}

Due to more radio access points (e.g., APs and SBSs) and wider coverage in dense mmWave cellular network, if the two-stage BFT mechanism for dense mmWave WLAN is applied directly in dense mmWave cellular network, it will cause a very large BFT overhead. Therefore, we propose a hierarchical three-stage BFT mechanism, which can adapt to the expansion of network scale. The proposed threestage BFT mechanism can establish the directional links between MBS and SBSs, between SBSs and APs, and between APs and UEs. Based on this BFT information, we also propose a BFT information-aided radio access resource management algorithm, which sets the priority of radio access resource allocation based on link energy efficiency. Compared with the scheme that sets the priority of radio access resource allocation according to link SNR (or link SINR), our scheme achieves better performance in terms of average energy efficiency and throughput per link.

\section{Data Availability}

The simulation data used to support the findings of this study are available from the corresponding author upon request.

\section{Conflicts of Interest}

The authors declare that there is no conflict of interest regarding the publication of this article.

\section{Acknowledgments}

This work was supported in part by the National Natural Science Foundation of China (No. 61873352 and No. 61803387).

\section{References}

[1] J. Tan, W. Liu, T. Wang, M. Zhao, A. Liu, and S. Zhang, "A high-accurate content popularity prediction computational modeling for mobile edge computing using matrix completion technology," Transactions on Emerging Telecommunications Technologies, no. article e3871, 2020.

[2] S. Huang, A. Liu, S. Zhang, T. Wang, and N. Xiong, "BD-VTE: a novel baseline data based verifiable trust evaluation scheme for smart network systems," IEEE Transactions on Network Science and Engineering, 2020.

[3] L. L. Wang, J. S. Gui, X. H. Deng, F. Zeng, and Z. Kuang, "Routing algorithm based on vehicle position analysis for internet of vehicle," IEEE Internet of Things Journal, 2020.

[4] T. Li, W. Liu, T. Wang, Z. Ming, X. Li, and M. Ma, "Trust data collections via vehicles joint with unmanned aerial vehicles in the smart Internet of things," Transactions on Emerging Telecommunications Technologies, no. article e3956, 2020.

[5] B. Jiang, G. Huang, T. Wang, J. Gui, and X. Zhu, "Trust based energy efficient data collection with unmanned aerial vehicle in edge network," Transactions on Emerging Telecommunications Technologies, no. article e3942, 2020.

[6] T. Li, A. Liu, N. N. Xiong, S. Zhang, and T. Wang, "A trustworthiness-based vehicular recruitment scheme for infor- mation collections in distributed networked systems," Information Sciences, vol. 545, pp. 65-81, 2021.

[7] J. An, K. Yang, J. Wu, N. Ye, S. Guo, and Z. Liao, "Achieving sustainable ultra-dense heterogeneous networks for $5 \mathrm{G}$," IEEE Communications Magazine, vol. 55, no. 12, pp. 84-90, 2017.

[8] N. Ye, X. Li, H. Yu, A. Wang, W. Liu, and X. Hou, "Deep learning aided grant-free NOMA toward reliable low-latency access in tactile Internet of things," IEEE Transactions on Industrial Informatics, vol. 15, no. 5, pp. 2995-3005, 2019.

[9] N. Ye, X. Li, H. Yu, L. Zhao, W. Liu, and X. Hou, "DeepNOMA: a unified framework for NOMA using deep multitask learning," IEEE Transactions on Wireless Communications, vol. 19, no. 4, pp. 2208-2225, 2020.

[10] J. Li, X. Li, A. Wang, and N. Ye, "Beamspace MIMO-NOMA for millimeter-wave broadcasting via full-duplex D2D communications," IEEE Transactions on Broadcasting, vol. 66, no. 2, pp. 545-554, 2020.

[11] Z. Li and J. Gui, "Energy-efficient resource allocation with hybrid TDMA-NOMA for cellular-enabled machine-tomachine communications," IEEE Access, vol. 7, pp. 105800105815, 2019.

[12] J. Gui, L. Hui, N. N. Xiong, and J. Wu, "Improving spectrum efficiency of cell-edge devices by incentive architecture applications with dynamic charging," IEEE Transactions on Industrial Informatics, p. 1, 2020.

[13] J. Gui, L. Hui, and X. Zhou, "Improving lifetime of cell-edge smart sensing devices by incentive architecture based on dynamic charging," IEEE Access, vol. 7, no. 1, pp. 7270372715, 2019.

[14] J. Gui, X. Dai, and X. Deng, "Stabilizing transmission capacity in millimeter wave links by Q-learning-based scheme," Mobile Information Systems, vol. 2020, Article ID 7607316, 17 pages, 2020.

[15] M. Xiao, S. Mumtaz, Y. Huang et al., "Millimeter wave communications for future mobile networks," IEEE Journal on Selected Areas in Communications, vol. 35, no. 9, pp. 19091935, 2017.

[16] Y. Liu, X. Fang, and M. Xiao, "Discrete power control and transmission duration allocation for self-backhauling dense mmWave cellular networks," IEEE Transactions on Communications, vol. 66, no. 1, pp. 432-447, 2018.

[17] W. Feng, Y. Wang, D. Lin, N. Ge, J. Lu, and S. Li, "When mmWave communications meet network densification: a scalable interference coordination perspective," IEEE Journal on Selected Areas in Communications, vol. 35, no. 7, pp. 1459$1471,2017$.

[18] P. Zhou, K. Cheng, X. Han et al., "IEEE 802.11ay-based mmWave WLANs: design challenges and solutions," IEEE Communications Surveys \& Tutorials, vol. 20, no. 3, pp. 1654-1681, 2018.

[19] P. Zhou, X. Fang, X. Wang, Y. Long, R. He, and X. Han, “Deep learning-based beam management and interference coordination in dense mmWave networks," IEEE Transactions on Vehicular Technology, vol. 68, no. 1, pp. 592-603, 2019.

[20] K. Cheng, X. Fang, and X. Wang, "Cloud based mmWave WLANs: architectural paradigms, proposals and perspectives," IEEE Wireless Communications, vol. 27, no. 1, pp. 170-177, 2020.

[21] P. Gallo, K. Kosek-Szott, S. Szott, and I. Tinnirello, "CADWAN: a control architecture for dense WiFi access networks," 
IEEE Communications Magazine, vol. 56, no. 1, pp. 194-201, 2018.

[22] A. Checko, H. L. Christiansen, Y. Yan et al., "Cloud RAN for mobile networks-a technology overview," IEEE Communications Surveys \& Tutorials, vol. 17, no. 1, pp. 405-426, 2015.

[23] J. Chen, B. Liu, H. Zhou, Q. Yu, L. Gui, and X. Shen, "QoSdriven efficient client association in high-density softwaredefined WLAN," IEEE Transactions on Vehicular Technology, vol. 66, no. 8, pp. 7372-7383, 2017.

[24] IEEE draft standard for information technology-telecommunications and information exchange between systems-local and metropolitan area networks-specific requirements-part 11: wireless LAN medium access control (MAC) and physical layer (PHY) specifications-amendment 7: enhanced throughput for operation in license-exempt bands above $45 \mathrm{GHz}$, IEEE Standard 802.11ay/Draft 2.0-2018, pp. 1-673, 2018.

[25] D. Tujkovic, K. Gomadam, N. Ahmed, M. Grigat, and C. Lange, "“Features for mmW distribution network use case," document IEEE 802.11, Facebook and Deutsche Telekom AG," 2017, https://mentor.ieee.org/802.11/dcn/17/1117-1321-00-00ay-features-for-mmw-distribution-networkuse-case.pdf.

[26] M. Giordani, M. Polese, A. Roy, D. Castor, and M. Zorzi, "Standalone and non-standalone beam management for 3GPP NR at mmWaves," IEEE Communications Magazine, vol. 57, no. 4, pp. 123-129, 2019.

[27] M. Giordani, M. Polese, A. Roy, D. Castor, and M. Zorzi, “A tutorial on beam management for 3GPP NR at mmWave frequencies," IEEE Communications Surveys \& Tutorials, vol. 21, no. 1, pp. 173-196, 2019.

[28] N. C. Luong, P. Wang, D. Niyato, Y. C. Liang, Z. Han, and F. Hou, "Applications of economic and pricing models for resource management in 5G wireless networks: a survey," IEEE Communications Surveys \& Tutorials, vol. 21, no. 4, pp. 3298-3339, 2019.

[29] Y. Liu, X. Fang, M. Xiao, and S. Mumtaz, "Decentralized beam pair selection in multi-beam millimeter-wave networks," IEEE Transactions on Communications, vol. 66, no. 6, pp. 27222737, 2018.

[30] Y. Fan, Z. Zhang, and H. Li, "Message passing based distributed learning for joint resource allocation in millimeter wave heterogeneous networks," IEEE Transactions on Wireless Communications, vol. 18, no. 5, pp. 2872-2885, 2019.

[31] W. Zhang, Y. Wei, S. Wu, W. Meng, and W. Xiang, "Joint beam and resource allocation in $5 \mathrm{G}$ mmWave small cell systems," IEEE Transactions on Vehicular Technology, vol. 68, no. 10, pp. 10272-10277, 2019.

[32] H. T. Nguyen, H. Murakami, K. Nguyen et al., "Joint user association and power allocation for millimeter-wave ultra-dense networks," Mobile Networks and Applications, vol. 25, no. 1, pp. 274-284, 2020.

[33] Q. Xue, X. Fang, and C. X. Wang, "Beamspace SU-MIMO for future millimeter wave wireless communications," IEEE Journal on Selected Areas in Communications, vol. 35, no. 7, pp. 1564-1575, 2017.

[34] A. Alkhateeb, S. Alex, P. Varkey, Y. Li, Q. Qu, and D. Tujkovic, "Deep learning coordinated beamforming for highly-mobile millimeter wave systems," IEEE Access, vol. 6, pp. 3732837348, 2018.

[35] P. Liu, J. Blumenstein, N. S. Perovic, M. di Renzo, and A. Springer, "Performance of generalized spatial modulation
MIMO over measured $60 \mathrm{GHz}$ indoor channels," IEEE Transactions on Communications, vol. 66, no. 1, pp. 133-148, 2018.

[36] Y. Cui, X. Fang, Y. Fang, and M. Xiao, "Optimal nonuniform steady mmWave beamforming for high-speed railway," IEEE Transactions on Vehicular Technology, vol. 67, no. 5, pp. 4350-4358, 2018.

[37] H. Ren and M. Q.-H. Meng, "Game-Theoretic Modeling of joint topology control and power scheduling for wireless heterogeneous sensor networks," IEEE Transactions on Automation Science and Engineering, vol. 6, no. 4, pp. 610-625, 2009.

[38] P. V. Amadori and C. Masouros, "Low RF-complexity millimeter-wave beamspace-MIMO systems by beam selection," IEEE Transactions on Communications, vol. 63, no. 6, pp. 2212-2223, 2015. 